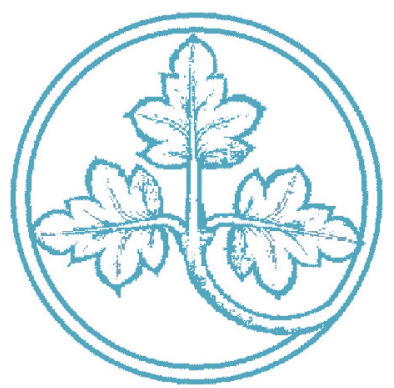

Information Processing in Decisions under Risk: Evidence for Compensatory Strategies based on Automatic Processes

Andreas Glöckner /

Ann-Katrin Herbold

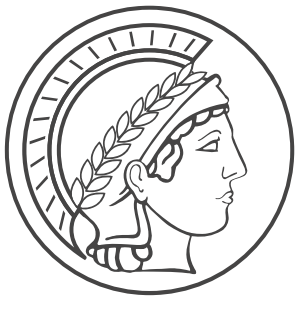




\title{
Information Processing in Decisions under Risk: Evidence for Compensatory Strategies based on Automatic Processes
}

\author{
Andreas Glöckner / Ann-Katrin Herbold
}

November 2008 


\title{
Information Processing in Decisions under Risk: Evidence for Compensatory Strategies based on Automatic Processes
}

\author{
Andreas Glöckner* / Ann-Katrin Herbold ${ }^{\ddagger}$
}

\begin{abstract}
Many everyday decisions have to be made under risk and can be interpreted as choices between gambles with different outcomes that are realized with specific probabilities. The underlying cognitive processes were investigated by testing six sets of hypotheses concerning choices, decision times, and information search derived from cumulative prospect theory, decision field theory, priority heuristic and parallel constraint satisfaction models. Our participants completed forty decision tasks of two gambles with two non-negative outcomes each. Information search was recorded using eye-tracking technology. Results for all dependent measures conflict with the prediction of the non-compensatory priority heuristic and indicate that individuals use compensatory strategies. Choice proportions are well predicted by a cumulative prospect theory. Process measures, however, indicate that individuals do not rely on deliberate calculations of weighted sums. Information integration processes seem to be better explained by models that partially rely on automatic processes such as decision field theory or parallel constraint satisfaction models.
\end{abstract}

Keywords: Risky Decisions, Cumulative Prospect Theory, Decision Field Theory, Priority Heuristic, Parallel Constraint Satisfaction, Eye Tracking, Intuition

Correspondence concerning this paper should be addressed to: Andreas Glöckner, Max Planck Institute for Research on Collective Goods, Kurt-Schumacher-Str. 10, D-53113 Bonn, Phone: +49-(0) 228 / 91416857 , E-mail: gloeckner@coll.mpg.de

$\ddagger \quad$ University Hospital, Bonn 
Risky decisions can be presented in the form of gambles comprising outcomes of different probability. According to classic expected utility models, individuals integrate outcomes and probabilities in a compensatory way and select the option with the highest weighted sum (Edwards, 1954; Savage, 1954; von Neumann \& Morgenstern, 1944). Cumulative prospect theory (CPT) (Kahneman \& Tversky, 1979; Tversky \& Kahneman, 1992), which was suggested as an alternative to these models, sticks with the assumption of compensatory integration and additionally postulates (amongst other things) that the evaluation of outcomes is dependent on a (flexible) reference point. CPT specifies that the value $\mathrm{V}$ of gambles is calculated by the weighted sum of subjective utilities and probabilities for all possible outcomes and defines a weighting function $\pi(\mathrm{pi})$ and a value function $\mathrm{v}(\mathrm{xi})$ that transform objective values $\mathrm{xi}$ and probabilities pi into subjective ones. In contrast to the assumption of weighted compensatory integration made by these models, Brandstätter, Gigerenzer, and Hertwig (2006) have argued that under certain conditions individuals might also use simple non-compensatory strategies (cf. Fishburn, 1974; Tversky, 1972). They proposed the priority heuristic (PH) as an alternative model for risky choices, which is based on the sequential non-compensatory comparison of "reasons" extracted from gambles. Several recent findings challenge the validity of the $\mathrm{PH}$ and support weighted compensatory models such as CPT (for results conflicting with both PH and CPT see Birnbaum, 2008b). Using different kinds of computer-based information-search paradigms (cf. Payne, Bettman, \& Johnson, 1988), it was found that in contrast to the predictions of the PH individuals search information mainly within gambles (Glöckner \& Betsch, 2008a, Exp. 3; Johnson, Schulte-Mecklenbeck, \& Willemsen, 2008), ${ }^{1}$ make choices that follow a weighted compensatory information integration (Birnbaum, 2008a; Birnbaum \& LaCroix, 2008; Glöckner \& Betsch, 2008a), and show decision times that increase with increasing similarity between the values $\mathrm{V}$ of the gambles (Glöckner \& Betsch, 2008a) as well as with increasing conflict between gambles (Hilbig, 2008). Furthermore, from a more general perspective, Rieskamp (in press) showed that probabilistic models predict risky choices better than deterministic ones and that (a probabilistic version of) DFT also outperformed a generalized version of the $\mathrm{PH}$.

Taken together, these findings challenge the PH as a process model and as a paramorphic ("asif") model (Hoffman, 1960). Although the results support compensatory models as paramorphic models, a closer inspection of the data corroborates the assumption that individuals might not apply them by deliberately calculating weighted sums. In decisions between two gambles, Glöckner and Betsch (2008a) observed an average decision time of 5.5 seconds and choices that mainly followed CPT. It is rather unlikely that participants conducted the necessary cognitive operations for the calculation of weighted sums deliberately (cf. elementary information processes, Lohse \& Johnson, 1996; Payne et al., 1988). It seems more likely that strategies based on partially automatic processes were used instead (Busemeyer \& Townsend, 1993; Glöckner \& Betsch, 2008b; Hammond, Hamm, Grassia, \& Pearson, 1987; Kahneman \& Frederick, 2002; Sloman, 2002).

1 For a different interpretation of the data by Johnson et al. (2008), see also Brandstätter, Gigerenzer, and Hertwig (2008). 
In the current study, we pursued two goals: First, we wanted to test whether the findings against the PH also hold if one uses a refined methodology and further dependent variables. In the studies testing the $\mathrm{PH}$, conflicting information search results have been captured using computerized information board paradigms in which information search is conducted by moving the mouse pointer to respective information cards. Recent evidence indicates that such a paradigm could influence decision strategies by limiting information search and hindering automatic processes (Glöckner \& Betsch, 2008c). Therefore, we intended to strengthen previous findings by readily presenting all pieces of information for the gambles on the screen and by recording information search with eye-tracking technology. Secondly, and more importantly, we aimed to improve our understanding of the processes underlying fast decision making under risk by testing several hypotheses (described in more detail later on) concerning information search derived from CPT, the PH, decision field theory (DFT) (Busemeyer \& Johnson, 2004; Busemeyer \& Townsend, 1993) and parallel constraint satisfaction models (PCS) (Glöckner \& Betsch, 2008b; Holyoak \& Simon, 1999; Thagard \& Millgram, 1995). Taking into account findings on the relation between levels of processing and fixation durations, we try to show that automatic processes play a major role in risky decision making. In the next section the decision models considered in this work are outlined. To enhance reading, an overview of all acronyms and key features for the considered theories is provided in Table 1. Note that some theories (i.e., the PH and CPT) differ in their predictions for the domains of gains and losses. To reduce complexity, our theoretical considerations focus on decisions between gambles with non-negative outcomes (i.e., gains) only.

Table 1. Acronyms and Key Features of Considered Theories

\begin{tabular}{lll}
\hline & \multicolumn{1}{c}{ Theories } \\
\hline Acronym & \multicolumn{1}{c}{ Name } & \multicolumn{1}{c}{ Key Features } \\
\hline CPT & $\begin{array}{l}\text { Cumulative Prospect } \\
\text { Theory }\end{array}$ & $\begin{array}{l}\text { Integration of subjective utility and subjective probability } \\
\text { according to a weighted sum } \\
\text { Non-compensatory consideration of single reasons (i.e., } \\
\text { minimum gain, probability of minimum gain, maximum } \\
\text { gain) }\end{array}$ \\
DFT & Priority Heuristic & $\begin{array}{l}\text { Evidence accumulation process; information sampling } \\
\text { proportional to outcome probabilities }\end{array}$ \\
PCS & Parallel Constraint Sat- & $\begin{array}{l}\text { Construction of mental representations and automatic } \\
\text { consistency maximizing by highlighting supporting evi- } \\
\text { dence for the favored option }\end{array}$ \\
\hline
\end{tabular}




\section{The Priority Heuristic}

According to $\mathrm{PH}$, individuals start decision making by screening all pieces of information. In this screening phase, the expected values of the gambles are roughly estimated and the $\mathrm{PH}$ is applied only if the expected values are similar (below a ratio of 1:2; Brandstätter et al., 2006). Aspiration levels (i.e., the minimum difference threshold between outcomes to consider them as being indeed different) are estimated by taking $1 / 10$ of the overall maximum outcome rounded to the nearest prominent numbers (i.e., all powers of 10 including their halves and doubles). If the PH is applied, individuals look up "reasons" without integrating probabilities and outcomes. First, individuals look up and compare the minimum outcomes of both gambles. If these outcomes differ by more than the aspiration level, the gamble with the higher minimum outcome is instantly selected. Otherwise, probabilities of the minimum outcomes are investigated. If they differ by more than .10 , the gamble with the lower probability for the minimum outcome is selected. If the difference in probabilities is smaller, the maximum outcomes are compared and the gamble with the higher one is selected.

Thus, according to the $\mathrm{PH}$, choices should follow a non-compensatory decision rule and decision times should increase with the number of reasons necessary to discriminate between options (Brandstätter et al., 2006). Information search should consist of an initial scanning, followed by a deliberate comparison of reasons. Hence, information search should be conducted between gambles by comparing the minimum outcomes (and in some cases also the probability of minimum outcomes).

\section{Weighted Compensatory Theories}

According to CPT (Kahneman \& Tversky, 1979; Tversky \& Kahneman, 1992), individuals select the gamble with the highest value $\mathrm{V}$ which is calculated by:

$$
V=\sum_{i} \pi\left(p_{i}\right) v\left(x_{i}\right)
$$

where the weighting function $\pi(\mathrm{pi})$ is an inverse S-shaped function of the probabilities pi and the value function v(xi) is an S-shaped function of the outcomes xi. The CPT proposes the following equations for both functions (for non-negative outcomes):

$$
v(x)=x^{\alpha}
$$

and

$$
\pi(p)=p^{\gamma} /\left(p^{\gamma}+(1-p)^{\gamma}\right)^{1 / \gamma}
$$

Tversky and Kahneman (1992) estimated the parameters $\alpha=0.88$ and $\gamma=0.61$. According to the theory, gambles (or prospects) are separately evaluated (Kahneman \& Tversky, 1979). Information should be sought within gambles and should be combined in a weighted additive manner according to equation 1 . To be able to derive process predictions, we interpret CPT as a process model (although it might be usually considered to be a paramorphic model). We assume that the 
weighted information integration is carried out in a stepwise manner that could be described by elementary information processes (i.e., specific information processing steps, Payne et al., 1988). Under this additional assumption, decision times should be rather high because the integration process (i.e., calculating weighted sums) is cognitively demanding and time-consuming. Furthermore, decision time should be equal for decision tasks with an equal number of outcomes since the number of necessary elementary information processes is equal. Information search could be expected to be carried out by a deliberate stepwise consideration of information within gambles (after the initial editing phase, Kahneman \& Tversky, 1979). Thus, information search should be equally distributed over all outcomes.

Another variant of compensatory theories is formalized in DFT (Busemeyer \& Johnson, 2004; Busemeyer \& Townsend, 1993). According to DFT, decisions are not made by calculating weighted sums but by a stochastic sequential sampling and evidence accumulation process. Specifically, in a stochastic process, outcomes of options (e.g., gambles) are repeatedly sampled with the probability of their realization. The affective reactions to the outcomes of each option are evaluated and compared. These comparisons are accumulated over time to form a preference state. If the preference state for one option reaches a threshold, this option is selected. Thus, DFT predicts choices according to weighted compensatory integration of subjective utilities and probabilities without relying on calculating weighted sums. Everything else being equal, decision time should decrease with increasing superiority of one option over the other and decisions should be made rather quickly by relying on automatic processes. Concerning the search for information, DFT predicts that outcomes are fixated proportionally to their probability and that the number of fixations increases with increasing probability of the respective outcome. Note that the probabilities of the outcomes of each gamble add up to one. Thus, according to DFT, both gambles should be inspected equally often. Furthermore, it could be expected that quick scanning of information predominates because information integration is based on automatic processes.

Another class of theories which predicts choices that approximate a weighted integration of probabilities and subjective utilities (without assuming that individuals calculate weighted sums) are PCS models. These models postulate that individuals form a mental representation of the decision task which can be modeled by interactive activation networks (Glöckner \& Betsch, 2008b; Holyoak \& Simon, 1999; McClelland \& Rumelhart, 1981; D. Simon, Krawczyk, \& Holyoak, 2004; Thagard, 1989). For decisions between two gambles with two (independent) outcomes, we suggest the network structure presented in Figure 1 (for similar models see also Glöckner, 2006, 2007, 2008; Glöckner \& Betsch, 2008b). The evaluation of options and outcomes are represented as nodes that differ in their validity. Relations between them are represented by links. Option nodes are connected to each other by a strong inhibitory link indicating that only one of them can be selected. Outcome nodes are connected to the options by links of different strength which represent the subjective probability of each outcome. Outcome nodes are connected with a general valuation node by excitatory (i.e., positive outcomes) or inhibitory (i.e., negative outcomes) links of different strength which represent the a priori valuation of the outcome. As soon as the tempo- 
rary network is constructed, activation spreads from the general valuation node which is simulated as an iterative updating mechanism (McClelland \& Rumelhart, 1981). These PCS processes operate towards maximizing consistency in the network by changing the activation of the nodes. Initial advantages of one or the other option (e.g., gamble) are accentuated by automatically highlighting supporting information (i.e., good outcomes for the favored option) and by devaluating contrary information (i.e., good outcomes for the non-favored option / bad outcomes for the favored option). As already mentioned, the models basically predict weighted compensatory information integration of subjective utilities and probabilities (Glöckner, 2006). ${ }^{2}$ Decision time should decrease with increasing superiority of one option over the other (which of course makes consistency maximizing easier), and decision times should be short because decisions are based on automatic processing. Information search should be focused more strongly on the favored option since the respective evidence is highlighted and given bigger weight in the decision. Scanning of information to construct a consistent mental representation should predominate in the decision.

Mathematically this is due to the fact that activation spreads from the general valuation node to the outcome nodes via links that represent subjective values and from the outcome nodes to the options nodes via links that represent subjective probabilities (see Figure 1) according to the following iterative activation function (McClelland \& Rumelhart, 1981):

$a_{i}(t+1)=a_{i}(t)(1-$ decay $)+\left\{\begin{array}{ccc}\text { if } & \text { input }_{i}<0 & \text { input }_{i}\left(a_{i}(t)-\text { floor }\right) \\ \text { if } & \text { input }_{i} \geq 0 & \text { input }_{i}\left(\text { ceiling }_{-} a_{i}(t)\right)\end{array}\right.$

ai $(\mathrm{t})$ represents the activation of the node $\mathrm{i}$ at iteration t. The parameters floor and ceiling stand for the minimum and maximum possible activation. Inputi(t) is the activation node $i$ receives at iteration $t$, which is computed by summing up all products of activations and connection weights wij for node $\mathrm{i}$.

$\operatorname{input}_{i}(t)=\sum_{j=1 \rightarrow n} w_{i j} a_{j}(t)$

Decay is a constant decay parameter. Activation is updated over a series of iterations $i$ until a stable solution is found which means that activation of all nodes reach an asymptotic level and the overall consistency in the model does not change anymore. To simplify the model, we use fix values for some of the free parameters in the model. Floor and ceiling are set to -1 and +1 , respectively, and a decay of .05 is used. The stability criterion used for terminating the process is 10 cycles with no energy changes bigger than $10^{-6}$. The only remaining individual-specific parameters are connection weights which are calculated from subjective probabilities and values using a monotonous transformation function. 


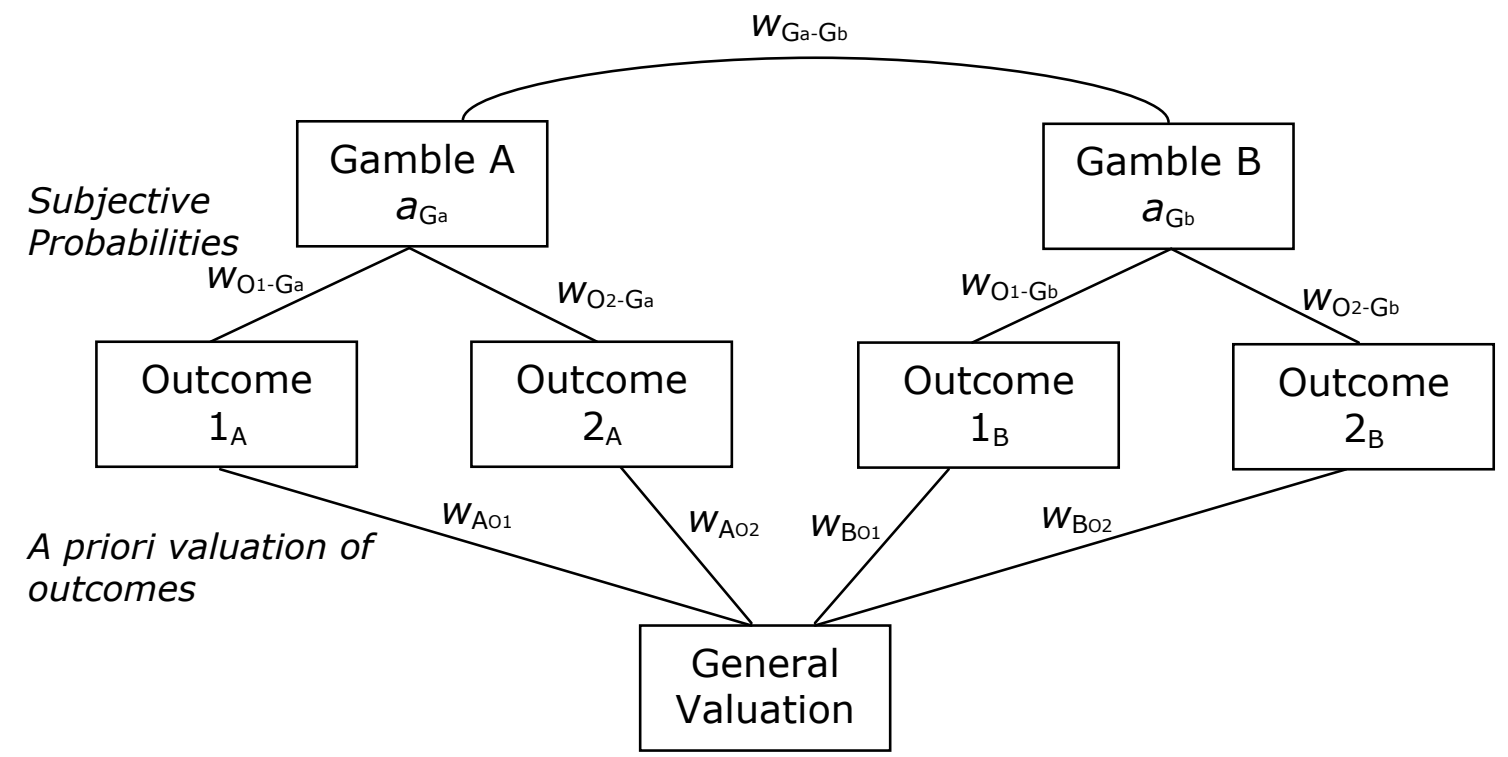

Figure 1. PCS network structure for decisions between two gambles with two independent outcomes each. The evaluation of gambles and outcomes are represented by nodes. The a priori valuations of outcomes are represented by links of outcomes to the general valuation node. The subjective probabilities of outcomes are represented by links between the outcome nodes and gamble nodes. Activation spreads along the network and node activations a are updated according to an iterative PCS process until a consistent and stable solution is found. The gamble with the higher final activation is chosen.

Overall, the following hypotheses will be tested (see also Table 7):

\section{Choice Hypotheses}

$\mathrm{H}_{1 \mathrm{a}}(\mathrm{PH})$ : Choices in decision tasks follow a non-compensatory integration rule.

$\mathrm{H}_{1 \mathrm{~b}}$ (CPT, DFT, PCS): Choices in decision tasks follow a weighted compensatory integration of subjective utilities/values and probabilities (i.e., expected utility / value $V$ ).

\section{Decision Time Hypotheses}

$\mathrm{H}_{2 \mathrm{a}}(\mathrm{PH})$ : Decision time increases with an increasing number of reasons that have to be considered.

$\mathrm{H}_{2 \mathrm{~b}}$ (DFT, PCS): Decision time decreases with increasing superiority concerning expected utility / value $V$ of one option over the other.

$\mathrm{H}_{2 \mathrm{c}}(\mathrm{CPT})$ : Decision times should be equal for all decision tasks. 


\section{Information Search Hypotheses}

$\mathrm{H}_{3 \mathrm{a}}(\mathrm{PH})$ : The amount of inspected information increases with an increasing number of reasons that have to be considered.

$\mathrm{H}_{3 \mathrm{~b}}(\mathrm{CPT})$ : The amount of inspected information is equal in all categories.

$\mathrm{H}_{3 \mathrm{c}}$ (DFT, PCS): The amount of inspected information increases with decreasing superiority concerning expected utility / value $V$ of one option over the other.

$\mathrm{H}_{4 \mathrm{a}}(\mathrm{PH})$ : Individuals start with initial screening and continue with a more thorough inspection of information.

$\mathrm{H}_{4 \mathrm{~b}}(\mathrm{CPT})$ : Individuals rely on a thorough sequential inspection of information.

$\mathrm{H}_{4 \mathrm{c}}$ (DFT, PCS): In the entire decision process, information search is mainly based on screening.

$\mathrm{H}_{5 \mathrm{a}}(\mathrm{PH})$ : Information is mainly sought by comparing outcomes between gambles (i.e., betweengamble transitions).

$\mathrm{H}_{5 \mathrm{~b}}(\mathrm{CPT})$ : Information is mainly sought within gambles (i.e., within-gamble transitions).

$\mathrm{H}_{5 \mathrm{c}}$ (DFT): Information is searched according to a random sampling process which leads to an equal number of within and between gamble transitions.

$\mathrm{H}_{6 \mathrm{a}}(\mathrm{PH})$ : Fixations are mainly focused on the minimum outcomes.

$\mathrm{H}_{6 \mathrm{~b}}(\mathrm{CPT})$ : Fixations are equally distributed over all outcomes.

$\mathrm{H}_{6 c}$ (DFT): Fixations are equally distributed over both gambles.

$\mathrm{H}_{6 \mathrm{~d}}$ (PCS): Fixations are concentrated on the outcomes of the favored gamble.

The decision time and information search hypotheses concerning CPT rest on the process assumptions that (a) information is integrated according to a deliberate serial process and that (b) computations are always carried out with the same speed. Both assumptions were imposed to make the theory testable and were not originally made by Tversky and Kahneman (1992). Throughout the paper we also do not consider the editing phase which was lined out in the original paper on prospect theory (Kahneman \& Tversky, 1979). Note that the editing phase could contain a screening process so that $\mathrm{CPT}$ also predicts $\mathrm{H}_{4 \mathrm{a}}$. The $\mathrm{PH}$ hypothesis concerning the dominance of between gamble transitions (i.e., $\mathrm{H}_{5 \mathrm{a}}$ ) can only be derived under the assumption that there are not more within than between gamble transitions in the screening phase. Because $\mathrm{PH}$ does not specify information search in the screening phase, we assume a random search. This would result in an equal distribution of within and between gamble transitions and hence allow deriving $\mathrm{H}_{5 \mathrm{a}}$. 


\section{Methodological Preliminaries}

To test our information search hypotheses we rely on an eye-tracking methodology which has been used to investigate processes in decision making since the 70ies (Lohse \& Johnson, 1996; Russo, 1978; Russo \& Rosen, 1975) (for reviews of eye tracking research in general, see Rayner, 1978, 1998). In contrast to other research tools like computer based information board paradigms (Payne et al., 1988) and think-aloud protocols (Montgomery \& Svenson, 1989; Russo, Johnson, $\&$ Stephens, 1989), eye-tracking has the advantage that it decreases the likelihood of influencing the decision process by the method itself. Process tracing methods such as information board paradigms sometime influence decision behavior (Billings \& Marcus, 1983; Maule, 1994) and might hinder participants from relying on automatic processing by constraining quick comparisons and information search (Glöckner \& Betsch, 2008c). Thus, eye-tracking studies provide more internally valid results particularly for decision tasks requiring information search in which information is readily available.

Not only does eye-tracking allow for tracing information search without hindering automatic processes, but it also has the additional advantage that fixation durations can be used to provide insights into cognitive processes. Specifically, Velichkovsky et al. have argued that fixation time increases with an increasing level of processing (Velichkovsky, 1999; Velichkovsky, Rothert, Kopf, Dornhofer, \& Joos, 2002). It has been shown that more superficial levels of processing such as screening are connected with relatively short fixations (up to $250 \mathrm{~ms}$ ), whereas deeper processing such as deliberate consideration of information is related with longer fixations $(>500$ ms). Velichkovsky, Challis, and Pomplun (1995) could for instance show that when comparing pictures, in an earlier phase of automatic information search and scanning short fixations prevail, whereas in a later phase, "when the crucial difference is about to be found, the fixation durations rise to $500 \mathrm{~ms}$ and more. [...] Obviously, this final phase of visual search can be attributed to some higher level of cognitive processing, which culminates in a conscious decision." (Velichkovsky, 1999, p. 214). Furthermore, in driving simulations, it could be shown that the appearance of a critical event leads to a decrease in short (pre-attentive scanning) fixations and an increase in long (attentive processing) fixations (Velichkovsky, Dornhoefer, Pannasch, \& Unema, 2001). In a similar vein, eye-tracking research on language processing has shown that more automatic processes such as silent reading are related with shorter fixations $(M=225 \mathrm{~ms})$ whereas more effortful processes such as typing lead to longer fixations $(M=400 \mathrm{~ms})$ (Rayner, 1998). Based on this literature, it seems reasonable to assume for this study that cognitive processes comprising conscious mathematical steps of information integration should go along with long fixations whereas scanning and automatic processes should produce mainly short fixations.

Consequently eye-tracking technology allows investigating whether individuals mainly scan information (and rely on automatic, intuitive processes of information integration) or whether they compute information on a higher level of attention (and use more thorough deliberate comparison of information). This allows testing our information search process hypotheses $\left(\mathrm{H}_{4 \mathrm{a}-\mathrm{c}}\right)$. In the analysis, we categorize short fixations $(<250 \mathrm{~ms})$ as connected with scanning and automatic operations, and longer fixations (>500 ms) as connected with deliberate operation. (Medium fixa- 
tions of 251 up to $500 \mathrm{~ms}$ are, of course, also reported but they are not used to test specific hypotheses.)

\section{Method}

\section{Participants and Design}

Eighteen students with different majors from the University of Bonn participated in the experiment which was part of a 1.5-hour experimental battery. Participants signed a consent form in which they were informed that they could quit the experiment any time. They were paid $€ 18$ for participation. We used similar procedures and materials as in previous studies (Glöckner \& Betsch, 2008a, Exp. 1 \& 3) except for the fact that this study was conducted using eye-tracking equipment. Participants completed 40 decision tasks which were manipulated using a withinsubjects design in which each respondent makes 8 similar decisions in each of the 5 categories of decision tasks. Examples for the five categories, their acronyms and key features are presented in Table 2.

Table 2. Acronyms and Key Features of the Decisions Tasks

\begin{tabular}{|c|c|c|}
\hline \multicolumn{3}{|c|}{ Categories of Decision Tasks } \\
\hline Acronym & Example & Key Features \\
\hline $\mathrm{CERT}_{\mathrm{PRO}}$ & $\begin{array}{l}\text { A: } .50, € 30 ; .50, € 40 \\
\text { B: . } 30, € 0 ; .70, € 50\end{array}$ & $\begin{array}{l}\text { One zero outcome for } B \text {; the certainty effect points pro } A \\
\text { and all theories predict mainly choices for } A\end{array}$ \\
\hline CERT $_{\text {CON }}$ & $\begin{array}{l}\text { A: } .40, € 0 ; .60, € 160 \\
\text { B: .60, } € 10 ; .40, € 220\end{array}$ & $\begin{array}{l}\text { One zero outcome for } A \text {; the certainty effect points contra } \\
\mathrm{A} ; \mathrm{PH} \text { predicts } \mathrm{A} \text {, all other theories predict } \mathrm{B}\end{array}$ \\
\hline SIM & $\begin{array}{l}\text { A: } .60, € 50 ; .40, € 60 \\
\text { B: } .40, € 40 ; .50, € 69\end{array}$ & $\begin{array}{l}\text { All outcomes are very similar; value according to CPT is } \\
\text { similar for both gambles }\end{array}$ \\
\hline$M D_{\text {ALM_CERT }}$ & $\begin{array}{l}\text { A: . } 90, € 55 ; .10, € 500 \\
\text { B: .01, } € 0 ; .99, € 100\end{array}$ & $\begin{array}{l}\text { A has a high and a low outcome; in B a medium outcome } \\
\text { is almost certain }\end{array}$ \\
\hline MED $_{\text {CERT }}$ & $\begin{array}{l}\text { A: . .98, } € 83 ; .02, € 100 \\
\text { B: . } 85, € 38 ; .15, € 340\end{array}$ & $\begin{array}{l}\text { A leads to a medium outcome with certainty, B is risky } \\
\text { and has a high and a low outcome }\end{array}$ \\
\hline
\end{tabular}

\section{Procedure and Materials}

The experiment was computerized. First, participants were familiarized with the decision task and instructed to select whichever of the two gambles they preferred (for the complete instructions, see appendix). Participants were instructed to make good decisions and to proceed as fast as possible.

Each decision started with a blank screen (4 seconds) followed by a fixation cross ( 1 second), which centered the attention in the middle of the screen. Next, the gambles were presented in an 
ellipsoid display which assured that information was equally distant from the initial fixation point (Figure 2). Information was sorted so that the information for one gamble was presented on the left and for the other on the right side. Participants selected one of the two gambles by pressing a key on the left ("y") or right ("m") side of the keyboard.

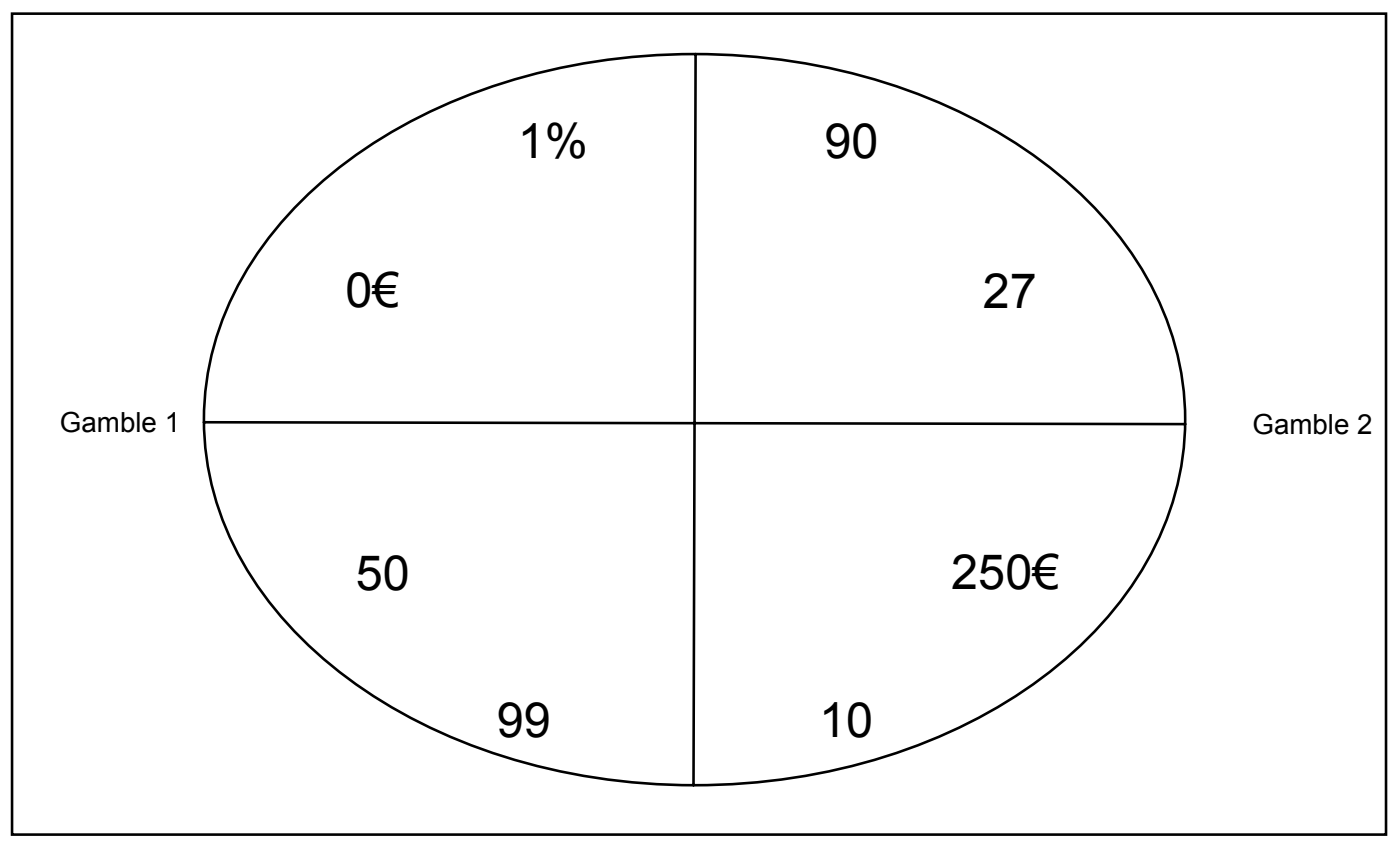

Figure 2. Decision tasks between gambles with two outcomes each. Left outcomes belong to gamble 1 , right outcomes belong to gamble 2 .

Eye movements were recorded using the Eyegaze binocular system (LC Technologies) with remote binocular sampling rate of $120 \mathrm{~Hz}$ and an accuracy of about $0.45^{\circ}$. The system is based on pupil-center/corneal reflection method to determine eye gaze. This method captures voluntary, saccadic eye movements that fixate a target object on fovea. An infrared-sensitive video camera, positioned below the computer monitor, observes the subject's eye and specialized image software generates $\mathrm{x}, \mathrm{y}$ coordinates for the gaze point on the monitor screen. Images were presented on a 17-inch color monitor (Samsung Synchmaster 740B, refresh rate $60 \mathrm{~Hz}$, reaction time 5ms) with a native resolution of $1280 \times 1024$. Viewed from a distance of $60 \mathrm{~cm}$, the screen subtended a visual angle of $28^{\circ}$ horizontally and $21^{\circ}$ vertically. Fixations were identified using a fixation radius of 20 pixels and a minimum fixation time of $50 \mathrm{~ms}$.

Choices, decision times, fixation coordinates and durations were recorded as dependent variables. We defined non-overlapping areas of interest (AOI) around each piece of information (i.e., outcome and probability) with the size of $170 \times 100$ pixels. The number and duration of fixations within each AOI were calculated. Furthermore, all direct transitions of eye-fixations between AOIs (i.e., direct movements of fixation from one AOI to another one) were determined. Fixations were categorized as short $(<250 \mathrm{~ms})$, medium $(251-500 \mathrm{~ms})$ or long ( $>500 \mathrm{~ms})$ fixations. As discussed above, short fixations below $250 \mathrm{~ms}$ are associated with scanning processes whereas more elaborated information processing is associated with long fixations of more than 500 ms (Velichkovsky, 1999; Velichkovsky et al., 2002). 
Forty decision tasks (see appendix) were shown in fixed randomized order. The presentation order of the gambles (i.e., gamble A first or gamble B first) and the order of outcomes within each gamble (i.e., low outcome first vs. high outcome first) were changed between trials to prevent gamble A from always being the gamble predicted by the PH. Each gamble had two nonnegative outcomes and the expected value for each pair of gambles was approximately equal. (Please note, that the (objective) expected value of a gamble $(E V=\Sigma v p)$ should not be equated with the (subjective) value $V$ of CPT.) Thus, the conditions for the application of the PH were ideal: individuals were instructed to decide fast, expected values did not differ between gambles, and it was possible to screen the information quickly without limiting information search by the experimental procedure. The PH was also given the advantage that its information search prediction (i.e., horizontal comparisons between gambles) was in line with the natural direction of reading (i.e., left to right). In contrast, the by CPT predicted search was not (i.e., vertical comparisons within gambles). The 40 presented decision tasks consisted of eight different versions of the five categories of decisions: CERT $_{\mathrm{PRO}}, \mathrm{CERT}_{\mathrm{CON}}$, SIM, MED $\mathrm{ALM}_{-} \mathrm{CERT}$ and MED $\mathrm{MERT}_{\mathrm{C}}$ (see Table 2).

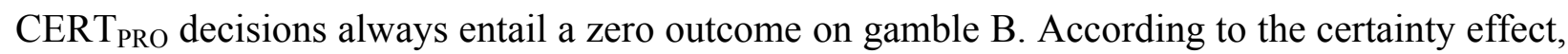
individuals should avoid this outcome and select gamble A. Thus, all strategies (PH, CPT, DFT and PCS) predict mainly choices for gamble A. CERT $_{\text {CON }}$ decisions entail a zero outcome on gamble A but the $\mathrm{PH}$ nevertheless predicts the selection of this gamble because the difference to the minimum outcome of gamble $B$ is small (i.e., below $1 / 10$ of the maximum outcome) and the probability of the minimum outcome for gamble B is higher. In both categories, the zerooutcome gamble has a considerably lower value $V$ (calculated according to CPT) than the other gamble. In the SIM decisions, both gambles have similar outcomes and the values $V$ of both gambles are roughly equal. Nevertheless, $\mathrm{PH}$ predicts all choices for gamble $\mathrm{A}$. In the $\mathrm{MED}_{\mathrm{ALM}} \mathrm{CERT}_{\mathrm{C}}$ and the $\mathrm{MED}_{\mathrm{CERT}}$ decision a medium outcome is almost certain (i.e., gamble A) or can be reached with certainty (i.e., gamble B). The respective other gamble consists of a high and a low outcome. Again PH predicts all decisions for gamble A, whereas the considered compensatory models predict choices for gamble A and B dependent on the parameters of the value and weighting function (for more details see Glöckner \& Betsch, 2008a). In summary, the PH predicts a very high proportion of choices for gamble A in all categories, whereas CPT and the other compensatory models predict a high proportion of choices for gamble A in CERT $\mathrm{PRO}_{\text {Peci- }}$ sions, a very low proportion in $\mathrm{CERT}_{\mathrm{CON}}$ decisions and a medium proportion in the remaining categories (Glöckner \& Betsch, 2008a, Table 1).

The $\mathrm{PH}$ predicts longer decision times in $\mathrm{CERT}_{\mathrm{CON}}$ decisions than in the remaining decisions because this category requires that at least two reasons have to be considered since the minimum gains do not differentiate according to the $\mathrm{PH}$. Although the expected values of the compared gambles were equal, the compared gambles differed in value $V$ according to CPT. In the SIM decision tasks the difference was small compared to the remaining categories. Hence, according to the weighted sum of subjective utilities and probabilities, the superiority of one over the other 
options was lower in the SIM decisions than in the remaining ones. PCS and DFT predict a higher decision time in this category compared to the other ones.

\section{Results}

\section{Choices and Decision Times}

Average choices and decision times (Figure 3) replicate very closely the pattern observed by Glöckner and Betsch (2008a). Aggregated choices are in line with the predictions of CPT and conflict with PH predictions. A $\chi^{2}$-test of independence between choices (Gamble A or B) and category turned out to be significant, $\chi^{2}(4, N=719)=118.5, p<.001$, indicating significant differences in choice proportions between categories. Thus, the PH-hypothesis $\mathrm{H}_{1 \mathrm{a}}$ has to be rejected. The proportion of choices for gamble A was no equally high for all categories. To test $\mathrm{H}_{1 b}$, it was investigated whether the proportion of choices for gamble $\mathrm{A}$ in the category $\mathrm{CERT}_{\mathrm{PRO}}\left(\mathrm{CERT}_{\mathrm{CON}}\right)$ was higher (lower) than in the remaining categories. Both tests turned out significant, $\chi^{2}(1$, $N=719)=77.4, p<.001$ and $\chi^{2}(1, N=719)=58.4, p<.001$, supporting $\mathrm{H}_{1 \mathrm{~b}}$ which states that aggregated choices are in line with choice proportions predicted by compensatory models based on subjective value and probability. On an individual level, choices were in 59\% of the tasks correctly predicted by $\mathrm{PH}$ and they were in $74 \%$ of the tasks in line with $\mathrm{CPT}^{3}$ which replicates the results by Glöckner and Betsch (2008a).
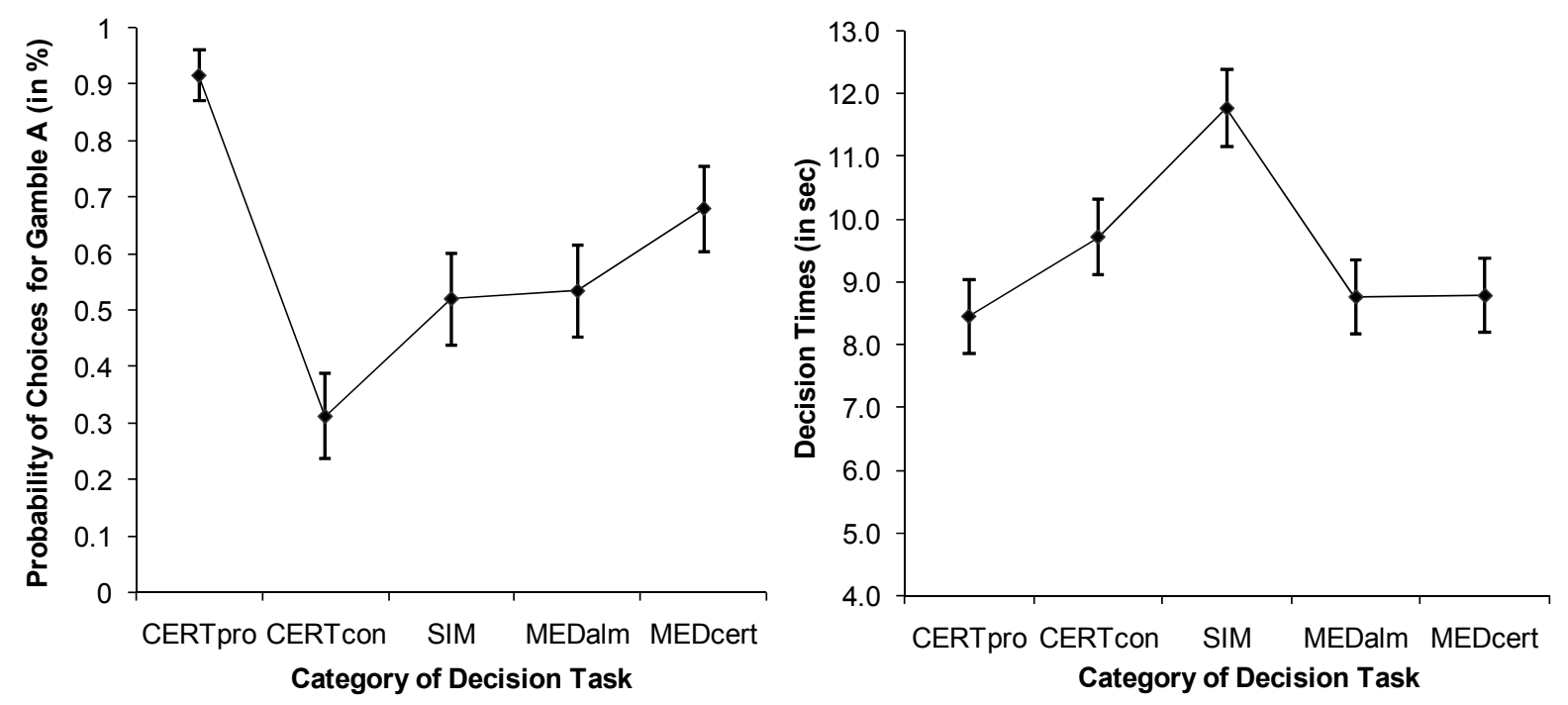

Figure 3. Choice proportions and decision times by category of decision task. Extreme decision time outliers are excluded $(M+/-3 S D)$. Error bars indicate .95 confidence intervals. Categories of decision tasks are described in Table 2.

3 We considered CPT without individual parameter fitting using the two sets of parameters determined in earlier work. Erev, Roth, Slonim, and Barron (2002) estimated the curvatures of the value function and the weighting function to be $\alpha=0.33$ and $\gamma=0.75$. Based on a different data set, Tversky and Kahneman (1992) estimated the parameters to be $\alpha=0.88$ and $\gamma=0.61$. For each participant the more predictive set was used. 
Decision times were analyzed using a repeated measurement analysis of variance (ANOVA) with category as within-participants factor and log-transformed decision times as dependent variable. There was a significant effect of category on decision time, $F(4,68)=15.2, p<.001$, $\eta^{2}=.43$. Thus, the CPT prediction $\left(\mathrm{H}_{2 \mathrm{c}}\right)$ that decision times are equal for all categories has to be rejected. The highest decision times were observed for the decision tasks in which the weighted sum of subjective values and probabilities of the options was relatively similar (i.e., SIM). Mean decision times excluding extreme outliers (i.e., $M+/-3 S D$ ) for the categories CERT $_{\text {PRO, CERT- }}$

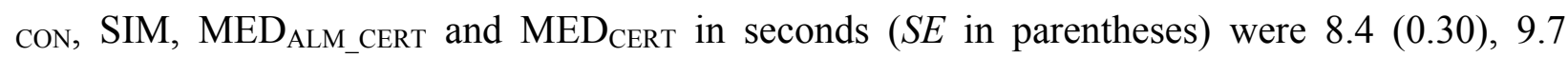
$(0.30), 11.8(0.31), 8.6(0.30)$, and 8.8 (0.30) (see also Figure 3, right). A contrast testing the PHhypothesis $\left(\mathrm{H}_{2 \mathrm{a}}\right)$ that decision times are higher for $\mathrm{CERT}_{\mathrm{CON}}$ than for the remaining categories (because only in this category the first reason does not differentiate between options) turned out to be not significant, $F(1,68)=0.67, p=.42$. The comparison between SIM and the remaining categories testing the DFT/PCS-hypothesis $\mathrm{H}_{2 \mathrm{~b}}$ was significant, $F(1,68)=54.0, p<.001$. Thus, choices are in line with the predictions by CPT, DFT, and PCS and decision times support DFT and PCS. Findings on both variables conflict with the predictions of the $\mathrm{PH}$.

Note that the mean decision time $(M=9.5 \mathrm{~s})$ was higher than in a previous study using the same set of decisions in a more conventional presentation format (Glöckner \& Betsch, 2008a, Exp. 1: $M=5.5 \mathrm{~s}$ ) but lower than in a study in which information search had to be conducted using the computer mouse (Glöckner \& Betsch, 2008a, Exp. 3: $M=12.1 \mathrm{~s}$ ). The difference between decision times for the standard line-format display and for the current study might be attributed to the fact that pieces of information were placed further apart from each other. The difference between the current study and the mouse-based information-search study indicates that eye-tracking might influence information search less than other information search paradigms (see also Glöckner \& Betsch, 2008c; Lohse \& Johnson, 1996). Note, however, that decision times seem to be merely rescaled by the paradigm and that the general pattern remains rather constant (i.e., there seem to be no interactions between research paradigm and dependent variables). This indicates that the presentation format did not induce shifts in decision strategies. Finally, it is worth mentioning that the eye-tracking technology used in the current study induced considerably less variability in decision times (cf. $S E$ within categories) as compared to the information-board paradigm (Glöckner \& Betsch, 2008a, Exp. 3), which can be considered as an additional advantage.

\section{Amount of Information Search (Fixations)}

Over all gambles, the average number of fixations for one decision was $M=36.4(S E=0.83)$ with $87 \%$ of the fixations being in the eight AOIs. Thus, fixations were highly systematic and in each decision each piece of information (i.e., probability or outcome) was fixated approximately 4 times. To test the CPT-hypothesis $\left(\mathrm{H}_{3 \mathrm{~b}}\right)$, which states that fixations are equally distributed over categories, a repeated-measurement ANOVA was calculated with category of decision task as factor and number of fixations in AOIs as dependent variable. We found a significant effect of 
category, $F(4,68)=12.5, p<.001, \eta^{2}=.42$. The mean fixations per category are shown in Figure 4. To test the PCS/DFT-hypothesis $\left(\mathrm{H}_{3 \mathrm{c}}\right)$ that there are more fixations in the category SIM than in the remaining categories, a contrast was calculated which turned out significant, $F(1,68)=$ 46.1, $p<.001$, supporting hypothesis $\mathrm{H}_{3 \mathrm{c}}$. The $\mathrm{PH}$-hypothesis $\left(\mathrm{H}_{3 \mathrm{a}}\right)$ was tested by comparing the amount of information search between the category $\mathrm{CERT}_{\mathrm{CON}}$ and the remaining categories. According to the $\mathrm{PH}$, there should be more information search in $\mathrm{CERT}_{\mathrm{CON}}$ decisions, which was not supported by the data, $F(1,68)=0.04, p=.84$.

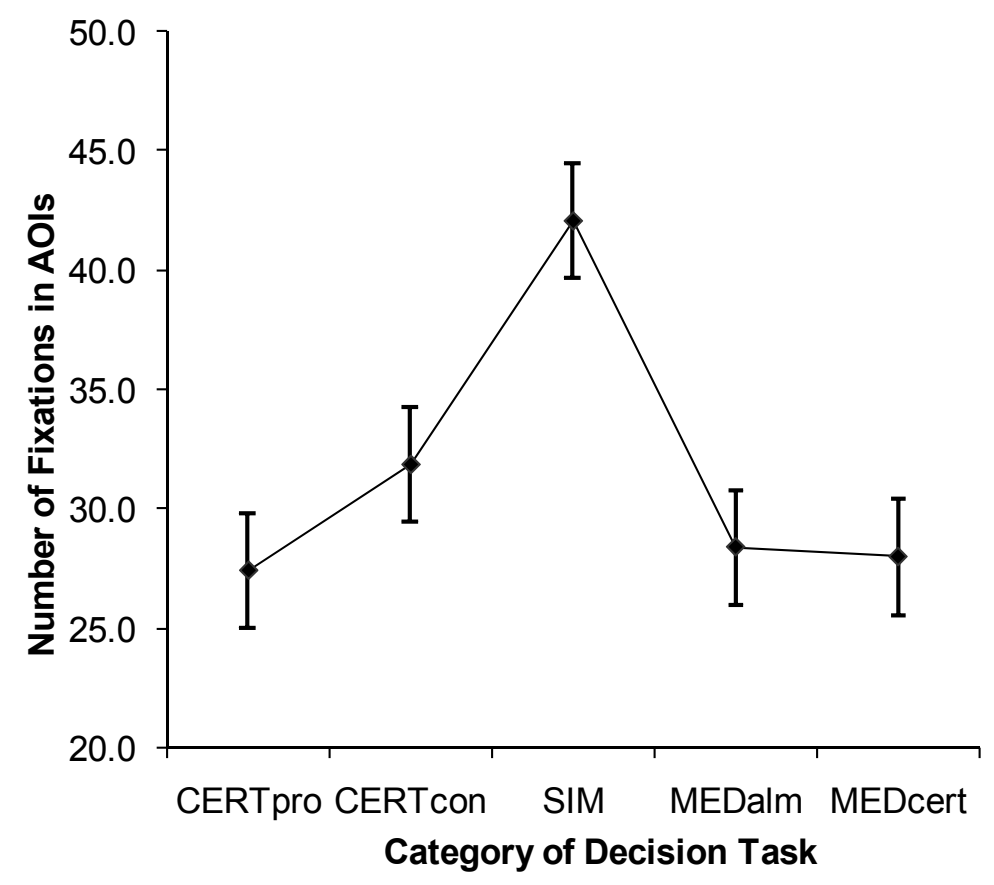

Figure 4. Mean number of fixations per category. Error bars indicate .95 confidence intervals. Categories of decision tasks are described in Table 2.

\section{Fixation Duration Analysis}

The PH postulates that individuals start by initially screening the pieces of information and later on compare pieces of evidence $\left(\mathrm{H}_{4 \mathrm{a}}\right)$. To test this initial screening hypothesis, individuals' fixations for each decision were analyzed. For pragmatic reasons, we considered the first 40 fixations only and divided them in blocks of 8 fixations (Fix_Block) each. (We used blocks of 8 fixations because 8 was the total amount of information available on the screen for each decision.) The first fixation on each decision screen was excluded from analysis because its position and length is likely to be biased by the previous fixation cross. ${ }^{4}$ Hence, Fix_Block 1 consisted of

4 The fixation cross was positioned in the middle of screen to assure that the initial fixation position was not biased in favor of single pieces of information. Exploratory data analysis nevertheless revealed that the first fixations often entailed a long fixation on the position of the fixations cross after it was removed. Usually, this fixation already started when the cross was still present. Since these fixations obviously did not represent information search of the decision process, we decided to remove all first fixations. The analyses concerning number of fixations and transitions were also calculated with and without first fixations which did not change results. 
fixations 2 to 9, Fix_Block 2 of the fixations 10 to 17 etc. To test the hypothesis that individuals start with initial screening, a $\chi^{2}$-test of independence between Fix_Block and fixation duration (short, medium, long) was calculated (taking into account Fix_Block 1 to 5). Short fixations $(<250 \mathrm{~ms})$ can be expected for screening processes, whereas long fixations $(>500 \mathrm{~ms})$ can be expected for deliberate comparisons of outcomes between gambles. The test turned out to be significant, $\chi^{2}(8, N=21178)=37.6, p<.001$. Nevertheless, inspection of the data (Figure 5, left) reveals that besides a general decrease of the number of fixations over time, the interaction effect was due to an increase in medium fixations (Block 1: 26.4\%; Block 5: 29.5\%) and a decrease in short (Block 1: 67.8\%; Block 5: 66.1\%) and long (Block 1: 5.8\%; Block 5: 4.4\%) fixations. Thus, we found a significant effect in the opposite direction as the one predicted by the initial screening hypothesis $\left(\mathrm{H}_{4 \mathrm{a}}\right)$. Our participants did not start by scanning all data and continued to compare values. In contrast, and in line with the PCS/CPT-hypothesis $\left(\mathrm{H}_{4 \mathrm{c}}\right)$ scanning (i.e., short fixations) remained the predominant information search strategy over the entire course of decision making accounting rather constantly for about $67 \%$ of the fixations. This result also contradicts the deliberate computation hypothesis $\left(\mathrm{H}_{4 \mathrm{~b}}\right)$ which states that long fixations should prevail.
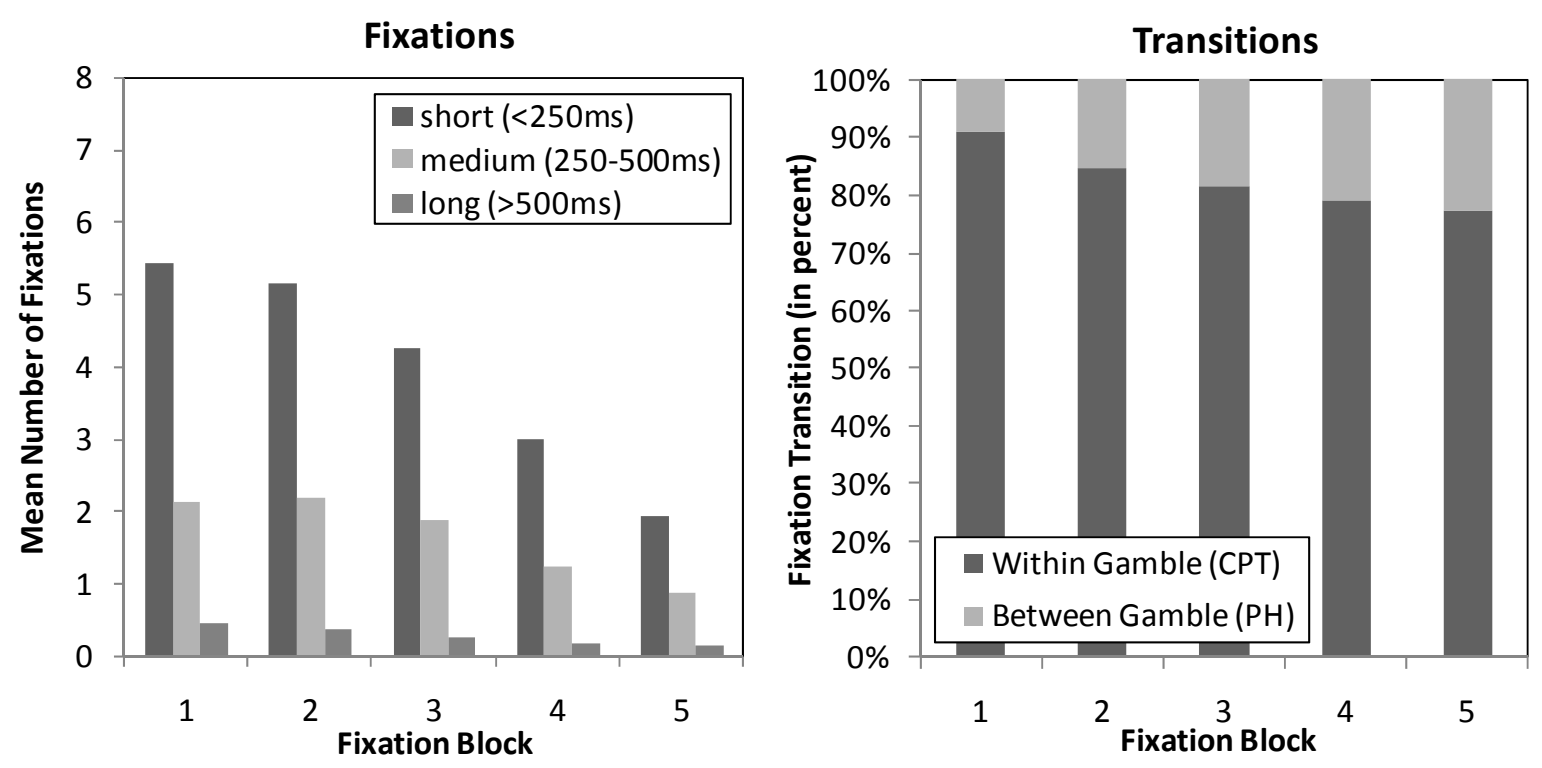

Figure 5. The development of long, medium and short fixations (left) and the proportion of direct within or between gamble transitions in fixations (right) in a decision. Each fixation block summarizes eight fixations excluding the first fixation (e.g., Block 1: fixations 2 to 9 , Block 2: fixations 10 to 17). 


\section{Information Search Process (Transitions)}

Overall there were 15,378 direct transitions of fixations between AOIs. Eighty-three percent of these transitions $(12,741)$ were within gambles and only $17 \%$ of the transitions turned out to be between gambles $(2,637)$. Thus, per decision, on average 17.4 within gamble transitions and 3.7 between gamble transitions were observed. This indicates that individuals, on average, looked up information gamble-wise and switched between gambles only 3 to 4 times. A $\chi^{2}$-test revealed that there were significantly more within-gambles transitions than between gamble transitions, $\chi^{2}(1, N=15378)=6638.8, p<.001$. Thus, the PH-hypothesis $\left(\mathrm{H}_{5 \mathrm{a}}\right)$ which states that information search is mainly conducted between gambles and the null hypothesis derived from DFT $\left(\mathrm{H}_{5 \mathrm{~b}}\right)$ that there is an equal distribution of between and within gamble transitions have to be rejected. ${ }^{5}$ Data support the CPT-hypothesis $\left(\mathrm{H}_{5 \mathrm{c}}\right)$ that information search is mainly conducted within gambles.

To investigate the development of transitions over time, we conducted a $\chi^{2}$-test of independence between Fix_Block and fixation transition (within gamble vs. between gamble). The test turned out significant, $\chi^{2}(4, N=12841)=196.7, p<.001$. Although being generally rather low, the number of between gamble fixation transitions increased over time indicating an increased amount of direct comparisons between gambles (Figure 5, right).

\section{Information Search Distribution (Attention)}

The level of attention that was given to certain aspects (i.e., outcomes, probabilities) of the gambles was measured by the number of fixations to the respective AOIs and by the overall fixation duration in these AOIs (Pomplun, Ritter, \& Velichkovsky, 1996). Note that decisions within each category of decision tasks have similar properties and therefore allow for an aggregated analysis. According to the $\mathrm{PH}$, attention should be focused on the minimum outcomes $\left(\mathrm{H}_{6 \mathrm{a}}\right)$. In contrast CPT predicts that attention should be equally distributed over all pieces of information $\left(\mathrm{H}_{6 \mathrm{~b}}\right)$. DFT predicts that attention should be proportional to the probabilities of the outcomes. Probabilities of the outcomes of each gamble add up to 1. Hence DFT predicts that the amount of information search for outcomes should not differ between gambles $\left(\mathrm{H}_{6 \mathrm{c}}\right)$. PCS predicts that attention is focused on the most favored option (and outcomes) because its advantages are highlighted $\left(\mathrm{H}_{6 \mathrm{~d}}\right)$.

To test these hypotheses, separate regressions were calculated for each category of decision tasks with the number of fixations in the AOIs as dependent variable and gamble, outcome and the interaction of both as predictors. To account for dependencies of observation because of the re-

$5 \quad$ Please note that the high amount of within gamble transitions makes it unlikely that the PH hypothesis was falsely rejected because of many within gamble transitions in the screening phase only (see the assumptions underlying H5a mentioned above). A complete screening of all pieces of information would at best (from the PH perspective) produce 6 within gamble transitions and 2 between gamble transitions. We observed on average almost three times as many within gamble transitions, which is even significantly higher than this maximum estimation, $\mathrm{t}(17)=7.87, \mathrm{p}<.001$. 
peated measurement design, standard errors were adjusted for clusters of dependent observations (i.e., participants) and robust standard errors were computed to account for violations of homoscedasticity. ${ }^{6}$ Regression coefficients are shown in Table 3. Descriptive statistics for outcomes and probabilities are presented in Figure 6.

Table 3. Regression for Number of Fixation

\begin{tabular}{lccccc}
\hline & $(1)$ & $(2)$ & $(3)$ & $(4)$ & $(5)$ \\
& CERTpro & CERTcon & SIM & MEDalm & MEDcert \\
\hline Gamble & $-1.472^{* * *}$ & $1.597^{* * *}$ & -0.347 & $-1.792^{* * *}$ & $-1.194^{* * *}$ \\
& $(-4.19)$ & $(6.07)$ & $(-0.87)$ & $(-5.29)$ & $(-6.85)$ \\
Outcome & & & & & \\
& 0.451 & $1.438^{* * *}$ & 0.0208 & $-0.868^{* *}$ & $-0.667^{*}$ \\
& $(1.74)$ & $(4.18)$ & $(0.12)$ & $(-3.38)$ & $(-2.39)$ \\
IE Gamble*Outcome & 0.0139 & -0.618 & 0.0833 & $3.549^{* * *}$ & 0.375 \\
& $(0.03)$ & $(-1.60)$ & $(0.30)$ & $(7.82)$ & $(1.29)$ \\
Constant & & & & & \\
& $4.424^{* * *}$ & $3.090^{* * *}$ & $6.368^{* * *}$ & $4.639^{* * *}$ & $5.097^{* * *}$ \\
\hline Observations & $(8.99)$ & $(9.58)$ & $(9.41)$ & $(11.23)$ & $(16.20)$ \\
\hline
\end{tabular}

Note. Regression with number of fixations in Areas of Interest (AOls) for outcomes (i.e., money) predicted by 'Gamble' $(A=0, B=1)$, 'Outcome' $\left(1^{\text {st }}=0,2^{\text {nd }}=1\right)$, and the interaction of both (IE Gamble $x$ Outcome). Regressions were conducted separately for the five categories of decision tasks (see Table 2). Analysis is based on 576 observations per category from 18 participants. To account for the repeated measurement design participants observations were clustered and robust standard errors were computed to account for violations of homoscedasticity. $t$ statistics are given in parentheses. Significance levels are indicated by ${ }^{*}$ $p<0.05,{ }^{* *} p<0.01,{ }^{* * *} p<0.001$.

The CPT-hypothesis $\left(\mathrm{H}_{6 \mathrm{~b}}\right)$ which states that information search is equally distributed over all outcomes and the DFT-hypothesis $\left(\mathrm{H}_{6 \mathrm{c}}\right)$ that information search is equally distributed over gambles were rejected for the categories $\mathrm{CERT}_{\mathrm{PRO}}, \mathrm{CERT}_{\mathrm{CON}}, \mathrm{MED}_{\mathrm{ALM}} \mathrm{CERT}_{\mathrm{C} T}$ and MED $\mathrm{MERT}_{\mathrm{C}}$. In all cases, there was a significantly stronger focus on the superior gamble (i.e., the gamble selected by the majority of participants), supporting the PCS-hypothesis $\left(\mathrm{H}_{6 \mathrm{~d}}\right)$. Note that in the remaining category SIM, no dominating option was available (i.e., both options were selected in about 50\% of the cases). Thus, no difference was predicted by PCS.

The PH-hypothesis $\left(\mathrm{H}_{6 \mathrm{a}}\right)$ that information search is mainly focused on the minimum outcomes is not supported by the data. The significant main effect for outcome in the $\mathrm{CERT}_{\mathrm{CON}}$ decisions and

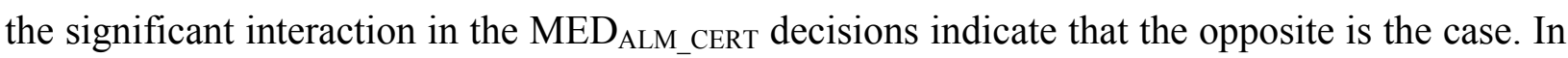

6 Standard errors were adjusted for clusters of repeated observations (i.e., dependence of error terms) using a random-effects model which allows for different correlations between error terms for each individual. HuberWhite robust standard errors were used to account for violations of the homoscedasticity assumption. Both are recommended standard functions of STATA and are implemented in supplementing regression commands by the options "cluster(id)" and "robust" (see Gould, Pitblado, \& Sribney, 2006; Hayes \& Cai, 2007). 
these gambles individuals focused significantly less often on the minimum outcomes, particularly on the zero outcomes.
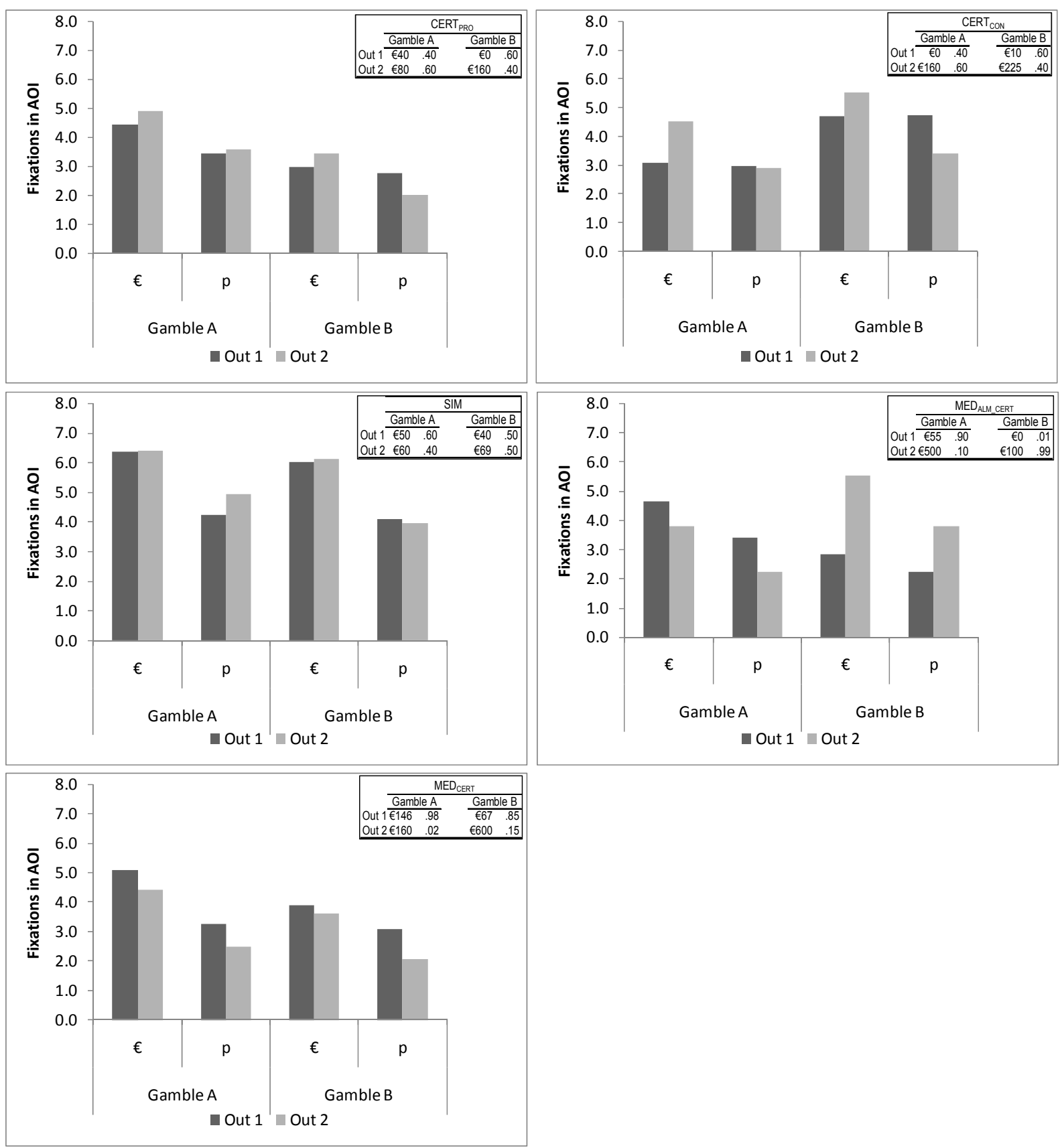

Figure 6. Fixations in Areas of Interest (AOls) for the five categories of decision tasks averaged over the eight different gambles in each category (see Table 2). One example is presented in the lower left corner of each graph. ' $€$ ' indicates the Euro-outcome of the gamble, ' $p$ ' indicates the probability of this outcome.

SE ranged from .11 to .39 with highest values in the SIM decisions.

To further test the hypotheses $\mathrm{H}_{6}$ we analyzed aggregated log-transformed fixation times per AOI. Again a regression was conducted with log-fixation times as criterion, gamble, outcome and the interaction as predictors and correction for clustered observations and heteroscedasticity (Table 4). The descriptive aggregated fixations time data are provided in Figure 7. 
Table 4: Regression for Fixation Time

\begin{tabular}{lccccc}
\hline & $\begin{array}{c}(1) \\
\text { CERTpro }\end{array}$ & $\begin{array}{c}(2) \\
\text { CERTcon }\end{array}$ & $\begin{array}{c}(3) \\
\text { SIM }\end{array}$ & $\begin{array}{c}(4) \\
\text { MEDalm }\end{array}$ & $\begin{array}{c}(5) \\
\text { MEDcert }\end{array}$ \\
\hline Gamble & $-0.214^{* * *}$ & $0.226^{* * *}$ & -0.0261 & $-0.238^{* *}$ & $-0.187^{* * *}$ \\
& $(-4.16)$ & $(4.90)$ & $(-0.88)$ & $(-3.59)$ & $(-4.41)$ \\
Outcome & & & & & \\
& 0.0512 & $0.176^{*}$ & 0.0355 & $-0.125^{*}$ & $-0.0911^{* *}$ \\
& $(1.45)$ & $(2.75)$ & $(1.63)$ & $(-2.60)$ & $(-3.13)$ \\
IE Gamble* Outcome & 0.00545 & -0.0722 & -0.0154 & $0.478^{* * *}$ & 0.0389 \\
& $(0.09)$ & $(-1.23)$ & $(-0.49)$ & $(6.70)$ & $(0.87)$ \\
Constant & & & & & \\
& $2.902^{* * *}$ & $2.698^{* * *}$ & $3.054^{* * *}$ & $2.890^{* * *}$ & $3.036^{* * *}$ \\
\hline Observations & $(48.89)$ & $(37.20)$ & $(58.56)$ & $(46.49)$ & $(99.44)$ \\
\hline
\end{tabular}

Note. Regression with log-transformed aggregated fixation times in Areas of Interest (AOls) for outcomes (i.e., money) predicted by 'Gamble' $(A=0, B=1)$, 'Outcome' $\left(1^{\text {st }}=0,2^{\text {nd }}=1\right)$, and the interaction of both (IE Gamble $x$ Outcome). Regressions were conducted separately for the five categories of decision tasks (see Table 2). Analysis is based on 576 observations per category from 18 participants. To account for the repeated measurement design participants, observations were clustered and robust standard errors were computed to account for violations of homoscedasticity. $t$ statistics are given in parentheses. Significance levels are indicated by ${ }^{*} p<0.05,{ }^{* *} p<0.01,{ }^{* * *} p<0.001$.

For the categories $\mathrm{CERT}_{\mathrm{PRO}}, \mathrm{CERT}_{\mathrm{CON}}, \mathrm{MED}_{\mathrm{ALM}}$ CERT and $\mathrm{MED}_{\mathrm{CERT}}$ the null hypothesis was rejected that fixation times were equally distributed over gambles $\left(\mathrm{H}_{6 \mathrm{~b}}\right.$ and $\left.\mathrm{H}_{6 c}\right)$. Findings support the PCS-hypothesis $\left(\mathrm{H}_{6 \mathrm{~d}}\right)$ that participants fixate longer on the favored gamble. Again, there was a strong additional effect in the $\mathrm{MED}_{\mathrm{ALM}}$ CERT $\operatorname{decisions}$ that the zero (or very low) outcome was focused much shorter than the remaining outcomes.

Fixation times did not support the $\mathrm{PH}$-hypothesis $\left(\mathrm{H}_{6 \mathrm{a}}\right)$ that information search is mainly focused on minimum outcomes. For the category $\mathrm{MED}_{\mathrm{ALM}} \mathrm{CERT}_{\mathrm{C}}$ the interaction turned out significant. The effect was mainly driven by a systematically lower fixation time for the minimum outcome of gamble B which is the opposite of what would be predicted by the PH-hypothesis $\left(\mathrm{H}_{6 \mathrm{a}}\right)$. 

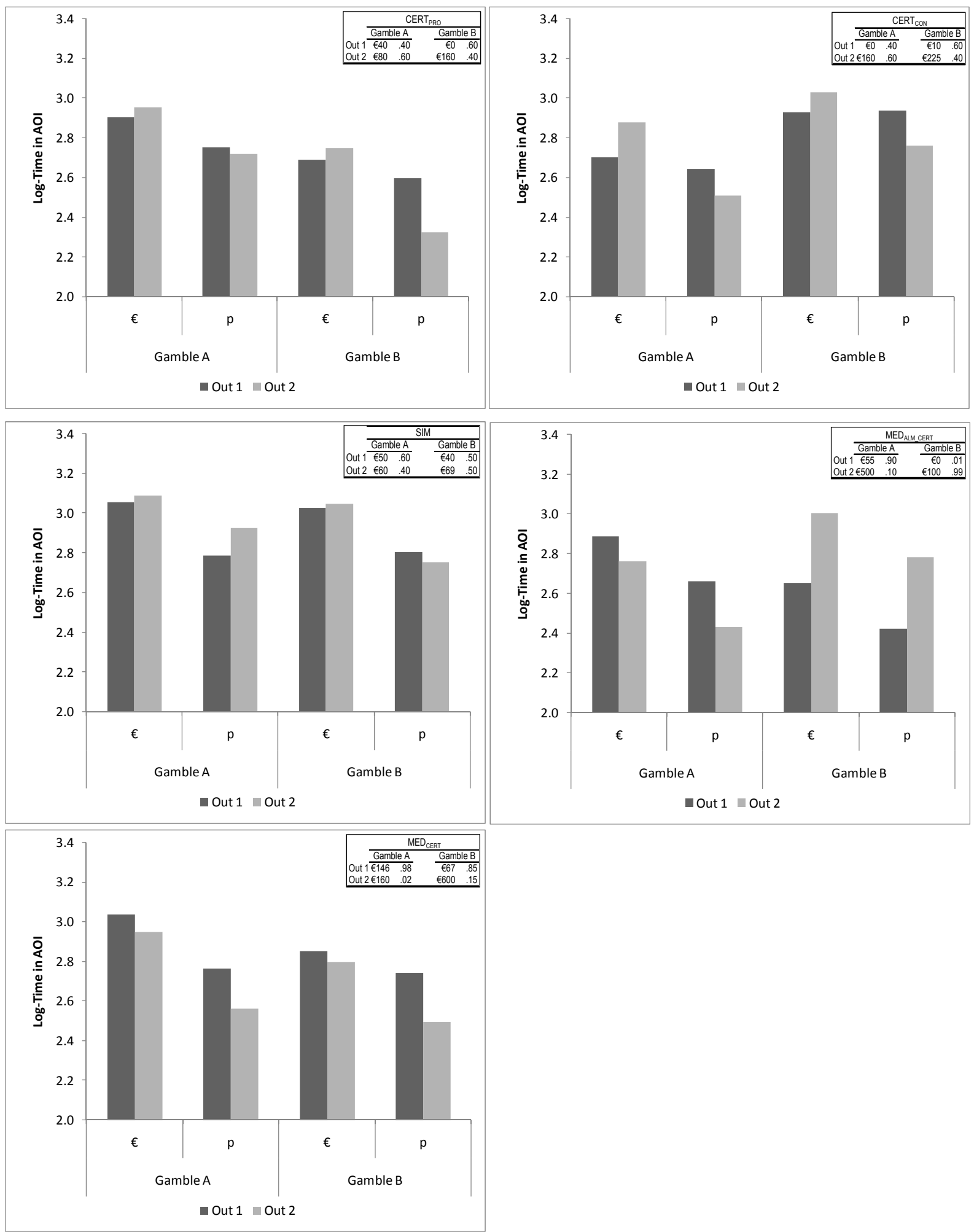

Figure 7. Log-transformed fixation time in Areas of Interest (AOIs) for the five categories of decision tasks averaged over the eight different gambles in each category (see Table 2). One example decision tasks is presented in the lower left corner of each graph. ' $E$ ' indicates the Euro-outcome of the gamble, ' $p$ ' indicates the probability of this outcome. SE ranged from .02 to .07 . 
To provide a more specific test for the PCS hypothesis $\mathrm{H}_{6 \mathrm{~d}}$, which states that participants mainly focus on the favored gamble, we conducted two regressions using number of fixations and fixation time as dependent variables and individuals' choices and the interaction of choices and gambles as additional predictors (Table 5). As predicted by PCS, the interaction of choice and gamble turned out highly significant in both regressions.

Table 5: Regression Analysis for Fixations Dependent on Choice

\begin{tabular}{lcc}
\hline & $(1)$ & $(2)$ \\
& Number of Fixations & Fixation Time \\
\hline Choice & $(0.000537$ & 0.0377 \\
& & $(1.69)$ \\
Gamble & $-0.988^{* * *}$ & $-0.149^{* * *}$ \\
& $(-6.10)$ & $(-6.90)$ \\
IE Choice*Gamble & $0.864^{* * *}$ & $0.153^{* * *}$ \\
& $(4.06)$ & $(6.20)$ \\
Outcome & 0.0751 & 0.00950 \\
& $(0.69)$ & $(0.47)$ \\
IE Gamble ${ }^{*}$ Outcome & $0.677^{* * *}$ & $0.0860^{* * *}$ \\
& $(5.20)$ & $(5.22)$ \\
Constant & $4.720^{* * *}$ & $2.893^{* * *}$ \\
& $(10.23)$ & $(59.34)$ \\
\hline Observations & 2876 & 2876 \\
\hline
\end{tabular}

Note. Regressions with number of fixations (1) and aggregated fixation times (2) in Areas of Interest (AOIs) for outcomes predicted by 'Choice' $(A=0, B=1)$, 'Gamble' $(A=0, B=1)$, 'Outcome' $\left(1^{\text {st }}=0,2^{\text {nd }}=1\right)$, and the interactions of Choice $\times$ Gamble and Gamble $x$ Outcome. Analysis is based on 2876 observations from 18 participants. To account for the repeated measurement design participants, observations were corrected for clusters and robust standard errors were computed to account for violations of homoscedasticity. $t$ statistics are given in parentheses. Significance levels are indicated by ${ }^{\star} p<0.05,{ }^{* \star} p<0.01,{ }^{* \star \star} p<$ 0.001 .

As can be seen in Figure 8, individuals focused attention on the gamble they selected. 


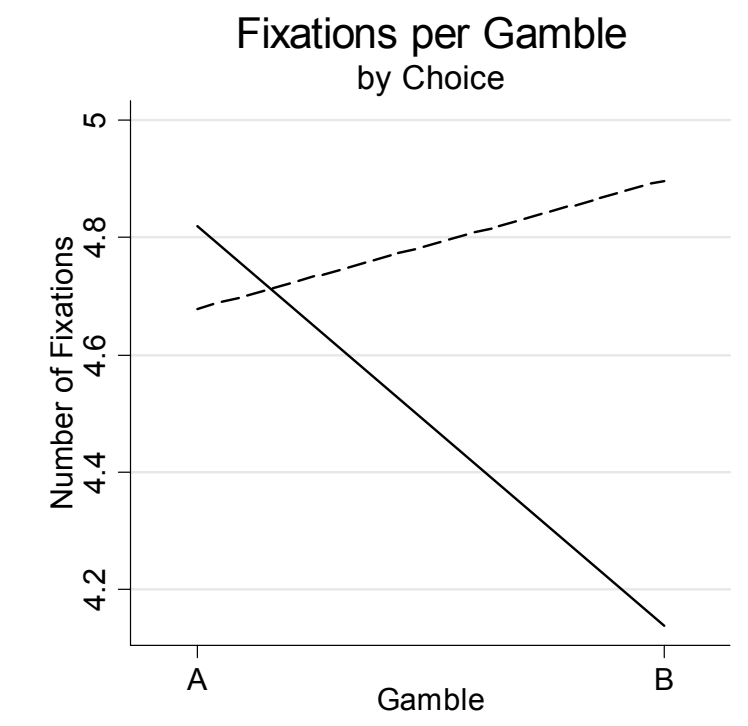

by Choice

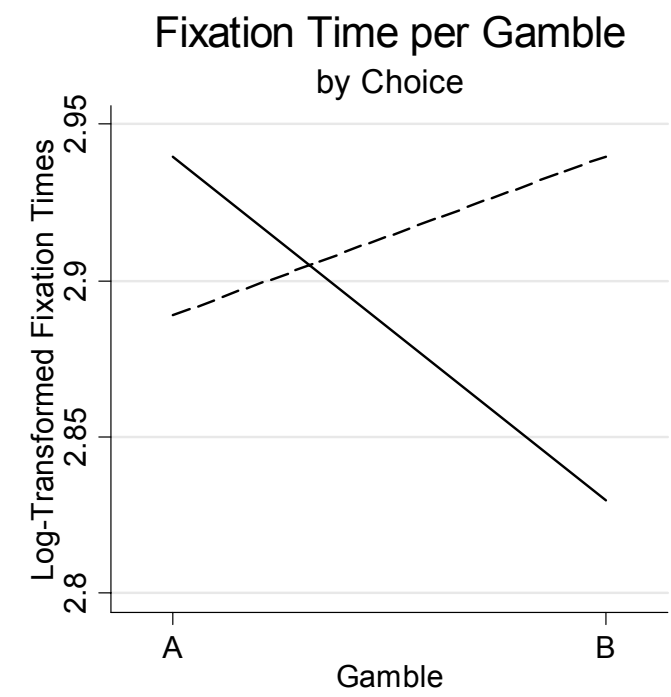

Figure 8. Number of fixations (left) and fixation times (right) per gamble by choice.

Furthermore, we tested the additional PCS prediction that individuals focus most strongly on particularly attractive outcomes (of the favored gambles) to increase their valuation. In the CERT $_{\text {PRO }}$ tasks, the second outcome of gamble $\mathrm{A}$ is particularly attractive because it pays a high amount of money and has a considerable probability. The same holds true for the second outcomes in gamble $\mathrm{B}$ in the $\mathrm{CERT}_{\mathrm{CON}}$ tasks. In the $\mathrm{MED}_{\mathrm{ALM}} \mathrm{CERT}$ the almost certain medium outcome (i.e., outcome 2) in gamble B is most attractive, and in the $\mathrm{MED}_{\mathrm{CERT}}$ tasks, the medium outcome with the high likelihood is attractive (Gamble A, outcome 1). In SIM decision tasks there is no particularly attractive outcome. For pragmatic reasons we selected the second outcome of gamble A which might be slightly more attractive to be included in the analysis. We compared the number of fixations to these most attractive outcomes with the fixations to the remaining outcomes within each gamble using single dummy coded variables as predictors (i.e., contrasts). As predicted by PCS, in all categories (except for SIM) the contrast turned out significant (Table 6). In each of the regressions the value of the constant represents the mean number of fixations for the not considered outcomes and the beta weight for the dummy variable indicates the additional fixations for the attractive outcome. 
Table 6: Regression for Fixation Frequency on most Attractive Outcomes

\begin{tabular}{|c|c|c|c|c|c|}
\hline & (1) & $(2)$ & (3) & $(4)$ & (5) \\
\hline Contrast & CERTpro & CERTcon & SIM & MEDalm & MEDcert \\
\hline $\begin{array}{l}\text { Gamble1 Out1 } \\
\text { vs. Others }\end{array}$ & & & & & $\begin{array}{l}1.116^{* * * x} \\
(7.56)\end{array}$ \\
\hline $\begin{array}{l}\text { Gamble1 Out2 } \\
\text { vs. Others }\end{array}$ & $\begin{array}{l}1.278^{*} \\
(2.87)\end{array}$ & & $\begin{array}{l}0.218 \\
(1.03)\end{array}$ & & \\
\hline $\begin{array}{l}\text { Gamble2 Out2 } \\
\text { vs. Others }\end{array}$ & & $\begin{array}{l}1.405^{* * *} \\
(5.50)\end{array}$ & & $\begin{array}{l}1.775^{* * *} \\
(8.82)\end{array}$ & \\
\hline Constant & $\begin{array}{l}3.597^{* * *} \\
(10.15)\end{array}$ & $\begin{array}{l}4.102^{* * *} \\
(10.56)\end{array}$ & $\begin{array}{c}6.171^{* \star *} \\
(8.75) \\
\end{array}$ & $\begin{array}{l}3.752^{* * *} \\
(13.87)\end{array}$ & $\begin{array}{l}3.981^{* * *} \\
(14.14)\end{array}$ \\
\hline Observations & 576 & 576 & 576 & 576 & 576 \\
\hline
\end{tabular}

Note. Regressions with frequency of fixations in Areas of Interest (AOIs) for outcomes predicted by dummy variables indicating 1 for the most attractive outcome in a category of gambles and 0 for the remaining outcomes (see Table 2). Analysis is based on 576 observations from 18 participants. $t$ statistics are given in parentheses. Significance levels are indicated by ${ }^{\star} p<0.05,{ }^{* \star} p<0.01,{ }^{* * *} p<0.001$.

An overview of the results concerning the hypotheses is provided in Table 7. Overall, all PH hypotheses were either not supported or rejected, which makes it unlikely that the $\mathrm{PH}$ is an appropriate process model for decision making. To derive process predictions, we interpreted CPT as a process model according to which options are selected by calculating weighted sums. Under this assumption, four of six CPT hypotheses were rejected. For the DFT, four out of six hypotheses were supported by the data. Thus, DFT seems to be at least partially a good model to describe outcomes and decision processes although it seems that information search is done more systematically than postulated by the proportional random sampling process of outcomes. For the PCS model five of six predictions were supported and for one variable no prediction was made. Thus, in our study outcome and process variables are explained best by this model. 
Table 7: Overview of Results

\begin{tabular}{|c|c|c|}
\hline Model & Hypothesis & Result \\
\hline \multicolumn{3}{|c|}{$\mathrm{H}_{1}$ : Choice proportion } \\
\hline $\mathrm{PH}$ & Non-compensatory integration rule & Rejected \\
\hline СРТ & Weighted compensatory integration of outcomes and probabilities & Supported \\
\hline DFT & Weighted compensatory integration of outcomes and probabilities & Supported \\
\hline PCS & Weighted compensatory integration of outcomes and probabilities & Supported \\
\hline \multicolumn{3}{|c|}{$\mathrm{H}_{2}$ : Decision time } \\
\hline $\mathrm{PH}$ & Increases with increasing number of reasons that have to be considered & Not Supported \\
\hline CPT & Equal & Rejected \\
\hline DFT & Decreases with increasing superiority of one option over the other & Supported \\
\hline PCS & Decreases with increasing superiority of one option over the other & Supported \\
\hline \multicolumn{3}{|c|}{$\mathrm{H}_{3}$ : Information search: amount of information } \\
\hline $\mathrm{PH}$ & Increases with number of reasons & Not Supported \\
\hline CPT & Equal & Rejected \\
\hline DFT & Increases with decreasing superiority of one option & Supported \\
\hline PCS & Increases with decreasing superiority of one option & Supported \\
\hline \multicolumn{3}{|c|}{$\mathrm{H}_{4}$ : Information search: screening vs. deeper processing } \\
\hline $\mathrm{PH}$ & Start with initial screening and continue with a more thorough inspection & Rejected \\
\hline CPT & Thorough sequential inspection of information & Rejected \\
\hline DFT & Mainly screening & Supported \\
\hline PCS & Mainly screening & Supported \\
\hline \multicolumn{3}{|c|}{$H_{5}$ : Information search: direction of transitions } \\
\hline $\mathrm{PH}$ & Between gambles & Rejected \\
\hline CPT & Within gambles & Supported \\
\hline DFT & Equal & Rejected \\
\hline PCS & No prediction & 1 \\
\hline \multicolumn{3}{|c|}{$\mathrm{H}_{6}$ : Information search: distribution of fixations / attention } \\
\hline $\mathrm{PH}$ & Mainly on minimum outcomes & Rejected \\
\hline CPT & Equal over all pieces of information & Rejected \\
\hline DFT & Equal over gambles & Rejected \\
\hline PCS & Focus on the favored gamble & Supported \\
\hline
\end{tabular}




\section{General Discussion}

The reported study shows that the recently proposed PH cannot account for choice behavior on the outcome level or on the processing level. Individuals do not seem to use the proposed noncompensatory decision rule in risky decisions. All PH-hypotheses concerning choices, decision time and information search were either not supported or directly rejected. Thus, our findings corroborate and extend previous investigations in a refined paradigm with open information presentation and with additional dependent measures derived from recording of eye-fixations.

We found that weighted compensatory models such as CPT, DFT and PCS can better account for individuals' choices. Decision times and the amount of information search increase with decreasing differences in expected utilities or value $V$ between gambles, even if the (objective) expected value is equal. This and other findings concerning information search (i.e., the predominant usage of short fixations and the unequal distribution of fixations over gambles) conflict with the idea that necessary calculations are deliberately conducted by computing weighted sums. Findings concerning fixation durations indicate that over the entire decision process quick screening processes predominate. This suggests that automatic processes, as indicated by DFT and PCS, might play a more important role in decision making than deliberate calculations or simple comparisons as proposed by non-compensatory heuristics (Gigerenzer \& Todd, 1999). Longer fixations which are related to more thorough information processing are less often used.

Against our findings, it might be argued that the predominance of short fixations was partially caused by the instruction to make good decisions and to proceed as fast as possible. Note, however, that it is usually assumed that increased time pressure leads to less information search and the application of non-compensatory strategies und should therefore increase application of PH (Edland \& Svenson, 1993; Payne et al., 1988; Rieskamp \& Hoffrage, 1999; Svenson \& Maule, 1993). According to these findings, individuals under time pressure should concentrate on a few (long) fixations. This is clearly not the case in our experiment. The current findings are in line with other work from our group which indicates that individuals, even under severe time pressure, use compensatory strategies based on automatic processing instead of switching to noncompensatory ones (Glöckner \& Betsch, 2008c) under the condition that the research paradigm does allow for quick information acquisition.

Interestingly, the proportion of long fixations decreases over time, which speaks against the hypothesis that more deliberate comparisons are used after an initial screening. Individuals seem to investigate information more thoroughly in the beginning and make relatively more medium length comparisons later on. In line with the prediction of CPT, information is clearly sought within gambles. Participants seem to evaluate each gamble separately and switch between gambles only a few times. This indicates that holistic impressions of each gamble are formed and compared. The level of attention to outcome information operationalized by the number of fixations and decision times is not equally distributed over gambles. Individuals focus more strongly on the favored options and on particularly attractive outcomes. This supports the PCShypotheses that a) the positive aspects of the favored option are highlighted by investigating 
them repeatedly and that $b$ ) particularly attractive outcomes (that usually speak for the preferred gamble) are highlighted and taken into account more strongly.

One of the central assumptions of DFT is that the calculation of weighted sums of outcomes and probabilities is circumvented by a stochastic sampling and evidence accumulation process. Our findings concerning the distribution of fixations (i.e., focus on favored gamble) and the direction of information search (i.e., search within gamble) speak against the validity of this assumption. Nevertheless, it might be argued that eye-fixations cannot be equated with instances of evidence accumulation. Individuals might investigate information once and retrieve them later on from memory. The fact that participants inspected pieces of information approximately four times and in a rather systematic manner, however, speaks against this interpretation. Furthermore, it can be expected that memory storage and retrieval for 8 pieces of information is cognitively effortful (cf. H. A. Simon, 1974). It is more likely that individuals relied on the less effortful strategy by looking up information directly. Although supporting DFT partially, our findings indicate that some of the assumptions of DFT, particularly predictions concerning stochastic information search, might have to be revised.

The PCS approach accounts well for the findings and seems to be a promising starting point for further investigations of decision processes in risky choices. Although there have been several attempts to apply PCS to decision making (Glöckner \& Betsch, 2008b; Holyoak \& Simon, 1999; D. Simon, Krawczyk et al., 2004; D. Simon, Snow, \& Read, 2004; Thagard \& Millgram, 1995), the PCS approach can be criticized for still being not sufficiently well specified. Up to now including the present study, mainly qualitative predictions on the aggregated level have been derived and tested (e.g., coherence shifts, decision times, choices, confidence). For further research, there is a need for testing more specific hypotheses on an individual level.

Furthermore, our results could be criticized in that the specific presentation format necessary for valid eye-recording might have influenced decision strategy and information search. It could for instance be argued that the comparison of probabilities was easier than the comparison between outcomes, because the former were presented closer to each other. Although this caveat has to be seriously considered, we argue that in eye-tracking studies the increases in costs for information search because of (minimal) increases in distance between pieces of information is rather low. Hence, such small changes should not influence decision strategies if it holds true that they are selected based on considerable learning experience (Rieskamp \& Otto, 2006). Moreover, as already mentioned above, our results concerning choice proportions and the pattern of decision times closely replicate the results observed with the same material in a study using the classic presentation format (Glöckner \& Betsch, 2008a, Exp. 1) which speaks against an influence of the presentation format on the decision strategy. Concerning our measures of information search we can, however, not completely rule out this possibility. The relation of transitions within vs. between gambles replicates results from a previous study using the same presentation format (Glöckner \& Betsch, 2008a, Exp. 3); compared to Johnson et al. (2008), however, we observed a higher proportion of within gamble transitions which might indicate a certain dependence of the results on presentation format. The observed difference can, however, not only be attributed to 
the presentation format; it could also be due to numerous other differences between the studies (e.g., structure of the selected tasks). A clarification of these issues shall be subject to further research.

In the current study, we investigated the processes underlying risky decisions by using an open information presentation format and eye-tracking technology. We extend previous research on decision process measurements by using fixation durations and relating them to information processing. In line with findings by Glöckner and Betsch (2008c), the results highlight the importance of automatic processes in decision making and the necessity for methods that do not hinder their application. Converging findings on other dependent variables (i.e., choices, decision times and other parameters of information search) provide evidence that the more elaborated analysis of fixation times leads to valid results. Nevertheless, further research with focus on methodological issues will be necessary to provide additional support for our conclusions.

Our results might be summarized as follows: to predict aggregated choice proportions in risky decisions with two gambles and two outcomes, CPT is a reasonably good (paramorphic) model. To account for the underlying processes and individual choice behavior, DFT and PCS models might be more suitable, but in DFT some of the assumptions concerning stochastic information search might have to be rethought. Simple non-compensatory models such as the PH seem to be less appropriate for predicting choices and describing processes. Overall, automatic processes seem to play an important role in risky decisions and a combination of open information presentation and eye-tracking seems to be a promising approach to investigate them. 


\section{References}

Billings, R. S., \& Marcus, S. A. (1983). Measures of compensatory and noncompensatory models of decision behavior: Process tracing versus policy capturing. Organizational Behavior \& Human Performance, 31(3), 331-352.

Birnbaum, M. H. (2008a). Evaluation of the priority heuristic as a descriptive model of risky decision making: Comment on Brandstatter, Gigerenzer, and Hertwig (2006). Psychological Review, 115(1), 253-260.

Birnbaum, M. H. (2008b). New tests of cumulative prospect theory and the priority heuristic: Probability-outcome tradeoff with branch splitting. Judgment and Decision Making, 3(4), 304-316.

Birnbaum, M. H., \& LaCroix, A. R. (2008). Dimension integration: Testing models without trade-offs. Organizational Behavior and Human Decision Processes, 105(1), 122-133.

Brandstätter, E., Gigerenzer, G., \& Hertwig, R. (2006). The Priority Heuristic: Making Choices Without Trade-Offs. Psychological Review, 113(2), 409-432.

Brandstätter, E., Gigerenzer, G., \& Hertwig, R. (2008). Risky choice with heuristics: Reply to Birnbaum (2008), Johnson, Schulte-Mecklenbeck, and Willemsen (2008), and Rieger and Wang (2008). Psychological Review, 115(1), 281-289.

Busemeyer, J. R., \& Johnson, J. G. (2004). Computational models of decision making. In D. J. Koehler \& N. Harvey (Eds.), Blackwell handbook of judgment and decision making (pp. 133-154). Malden, MA: Blackwell Publishing.

Busemeyer, J. R., \& Townsend, J. T. (1993). Decision field theory: A dynamic-cognitive approach to decision making in an uncertain environment. Psychological Review, 100(3), 432459.

Edland, A., \& Svenson, O. (1993). Judgment and decision making under time pressure: Studies and findings. In O. Svenson \& A. J. Maule (Eds.), Time pressure and stress in human judgment and decision making (pp. 27-40). New York, NY: Plenum Press.

Edwards, W. (1954). The theory of decision making. Psychological Bulletin, 51(4), 380-417.

Erev, I., Roth, A. E., Slonim, R. L., \& Barron, G. (2002). Combining a theoretical prediction with experimental evidence to yield a new prediction: An experimental design with a random sample of tasks. Unpublished manuscript. Columbia University and Faculty of Industrial Engineering and Management, Techion, Haifa, Israel.

Fishburn, P. C. (1974). Lexicographic orders, utilities, and decision rules: A survey. Management Science, 20, 1442-1472.

Gigerenzer, G., \& Todd, P. M. (1999). Simple heuristics that make us smart. Evolution and cognition. New York, NY: Oxford University Press.

Glöckner, A. (2006). Automatische Prozesse bei Entscheidungen [Automatic processes in decision making]. Hamburg, Germany: Kovac.

Glöckner, A. (2007). Does intuition beat fast and frugal heuristics? A systematic empirical analysis. In H. Plessner, C. Betsch \& T. Betsch (Eds.), Intuition in judgment and decision making (pp. 309-325). Mahwah, NJ: Lawrence Erlbaum. 
Glöckner, A. (2008). Shifting the bounds of rationality: The efficient interaction of automatic and deliberate processes in decision making. Paper presented at the CEREB Small Group Meeting Automaticity in Judgment and Decision Making.

Glöckner, A., \& Betsch, T. (2008a). Do people make decisions under risk based on ignorance? An empirical test of the Priority Heuristic against Cumulative Prospect Theory. Organizational Behavior and Human Decision Processes, 107(1), 75-95.

Glöckner, A., \& Betsch, T. (2008b). Modeling option and strategy choices with connectionist networks: Towards an integrative model of automatic and deliberate decision making. Judgment and Decision Making, 3(3), 215-228.

Glöckner, A., \& Betsch, T. (2008c). Multiple-reason decision making based on automatic processing. Journal of Experimental Psychology: Learning, Memory, and Cognition, 34(5), $1055-1075$.

Gould, W., Pitblado, J., \& Sribney, W. (2006). Maximum Likelihood Estimation with Stata (3rd ed.). College Station, TX: Stata Press.

Hammond, K. R., Hamm, R. M., Grassia, J., \& Pearson, T. (1987). Direct comparison of the efficacy of intuitive and analytical cognition in expert judgment. IEEE Transactions on Systems, Man, \& Cybernetics, 17(5), 753-770.

Hayes, A. F., \& Cai, L. (2007). Using heteroskedasticity-consistent standard error estimators in OLS regression: An introduction and software implementation. Behavior Research Methods, 39(4), 709-722.

Hilbig, B. E. (2008). One-reason decision making in risky choice? A closer look at the priority heuristic. Judgment and Decision Making, 3(6), 457-462.

Hoffman, P. J. (1960). The paramorphic representation of clinical judgment. Psychological Bulletin, 57(2), 116-131.

Holyoak, K. J., \& Simon, D. (1999). Bidirectional reasoning in decision making by constraint satisfaction. Journal of Experimental Psychology: General, 128(1), 3-31.

Johnson, E. J., Schulte-Mecklenbeck, M., \& Willemsen, M. C. (2008). Process models deserve process data: Comment on Brandstatter, Gigerenzer, and Hertwig (2006). Psychological Review, 115(1), 263-272.

Kahneman, D., \& Frederick, S. (2002). Representativeness revisited: Attribute substitution in intuitive judgment. In T. Gilovich, D. Griffin \& D. Kahneman (Eds.), Heuristics and biases: The psychology of intuitive judgment (pp. 49-81). New York, NY: Cambridge University Press.

Kahneman, D., \& Tversky, A. (1979). Prospect theory: An analysis of decision under risk. Econometrica, 47, 263-292.

Lohse, G. L., \& Johnson, E. J. (1996). A comparison of two process tracing methods for choice tasks. Organizational Behavior and Human Decision Processes, 68(1), 28-43.

Maule, A. J. (1994). A componential investigation of the relation between structural modelling and cognitive accounts of human judgement. Acta Psychologica, 87(2-3), 199-216.

McClelland, J. L., \& Rumelhart, D. E. (1981). An interactive activation model of context effects in letter perception: I. An account of basic findings. Psychological Review, 88(5), 375-407. 
Montgomery, H., \& Svenson, O. (1989). A think-aloud study of dominance structuring in decision processes. In H. Montgomery \& O. Svenson (Eds.), Process and structure in human decision making (pp. 135-150). Oxford, England: John Wiley \& Sons.

Payne, J. W., Bettman, J. R., \& Johnson, E. J. (1988). Adaptive strategy selection in decision making. Journal of Experimental Psychology: Learning, Memory, and Cognition, 14(3), 534-552.

Pomplun, M., Ritter, H., \& Velichkovsky, B. (1996). Disambiguating complex visual information: Towards communication of personal views of a scene. Perception, 25(8), 931-948.

Rayner, K. (1978). Eye movements in reading and information processing. Psychological Bulletin, 85(3), 618-660.

Rayner, K. (1998). Eye movements in reading and information processing: 20 years of research. Psychological Bulletin, 124(3), 372-422.

Rieskamp, J. (in press). Probabilistic nature of preferential choice. Journal of Experimental Psychology: Learning, Memory, and Cognition.

Rieskamp, J., \& Hoffrage, U. (1999). When do people use simple heuristics, and how can we tell? In Simple heuristics that make us smart (pp. 141-167). New York, NY: Oxford University Press.

Rieskamp, J., \& Otto, P. E. (2006). SSL: A Theory of How People Learn to Select Strategies. Journal of Experimental Psychology: General, 135(2), 207-236.

Russo, J. E. (1978). Eye fixations can save the world: A critical evaluation and a comparison between eye fixations and other information processing methodologies. In H. K. Hunt (Ed.), Advances in Consumer Research (pp. 561-570). Ann Arbor, MI: Association for Consumer Research.

Russo, J. E., Johnson, E. J., \& Stephens, D. L. (1989). The validity of verbal protocols. Memory \& Cognition, 17(6), 759-769.

Russo, J. E., \& Rosen, L. D. (1975). An eye fixation analysis of multialternative choice. Memory \& Cognition, 3(3), 267-276.

Savage, L. J. (1954). The foundations of statistics (2nd ed.). New York: Dover.

Simon, D., Krawczyk, D. C., \& Holyoak, K. J. (2004). Construction of Preferences by Constraint Satisfaction. Psychological Science, 15(5), 331-336.

Simon, D., Snow, C. J., \& Read, S. J. (2004). The Redux of Cognitive Consistency Theories: Evidence Judgments by Constraint Satisfaction. Journal of Personality and Social Psychology, 86(6), 814-837.

Simon, H. A. (1974). How big is a chunk? Science, 183(4124), 482-488.

Sloman, S. A. (2002). Two systems of reasoning. In T. Gilovich, D. Griffin \& D. Kahneman (Eds.), Heuristics and biases: The psychology of intuitive judgment (pp. 379-396). New York, NY: Cambridge University Press ISBN 0-521-79260-6 (hardcover); 0-521-79679-2 (paperback) Cambridge University Press Print.

Svenson, O., \& Maule, A. J. (1993). Time pressure and stress in human judgment and decision making. Time pressure and stress in human judgment and decision making. xxii, $335 \mathrm{pp}$. New York, NY: Plenum Press. 
Thagard, P. (1989). Explanatory coherence. Behavioral and Brain Sciences, 12(3), 435-502.

Thagard, P., \& Millgram, E. (1995). Inference to the best plan: A coherence theory of decision. In A. Ram \& D. B. Leake (Eds.), Goal-driven learning (pp. 439-454). Cambridge, MA: MIT Press.

Tversky, A. (1972). Elimination by aspects: A theory of choice. Psychological Review, 79(4), 281-299.

Tversky, A., \& Kahneman, D. (1992). Advances in prospect theory: Cumulative representation of uncertainty. Journal of Risk and Uncertainty, 5, 297-323.

Velichkovsky, B. M. (1999). From levels of processing to stratification of cognition: Converging evidence from three domains of research. In B. H. Challis \& B. M. Velichkovsky (Eds.), Stratification in cognition and consciousness (pp. 203-235). Amsterdam, Netherlands: John Benjamins Publishing Company.

Velichkovsky, B. M., Challis, B. H., \& Pomplun, M. (1995). Working memory and work with memory: Visuospatial and further components of processing. Zeitschrift fur Experimentelle Psychologie, 42(4), 672-701.

Velichkovsky, B. M., Dornhoefer, S. M., Pannasch, S., \& Unema, P. J. A. (2001). Visual fixations and level of attentional processing. In A. Duhowski (Ed.), Proceedings of the Symposium on Eye Tracking Research \& Applications (pp. 79-85). Palm Beach Gardens, NY: ACM Press.

Velichkovsky, B. M., Rothert, A., Kopf, M., Dornhofer, S. M., \& Joos, M. (2002). Towards an express-diagnostics for level of processing and hazard perception. Transportation Research Part F: Traffic Psychology and Behaviour, 5(2), 145-156.

von Neumann, J., \& Morgenstern, O. (1944). Theory of games and economic behavior (1st ed.). Princeton, NJ: Princeton University Press. 


\section{Appendix}

\section{Instruction}

You will be repeatedly presented with pairs of gambles. You should decide which of the gambles you would prefer to play. Each gamble has exactly two possible outcomes, A and B, which have some given probability of coming about. Each outcome leads to a certain monetary gain, which is given in Euro. [Example omitted] This means that in gamble 1 you have a 30\% chance of winning $€ 10$ and a $70 \%$ chance of winning $€ 20$, and so on. Your task is to select the gamble you prefer. Press " $y$ " to select gamble 1 and press " $m$ " to select gamble 2. [page break] If you have any questions, please ask the experimenter; otherwise press continue [page break] Please note: try to make accurate decisions and to proceed as quickly as possible. Put the left finger on " $y$ " and the right finger on "m" and press both keys to start the decision phase.

\section{Decision Tasks}

Table A1 shows the 40 decision tasks used in our experiment. Each decision task consists of two gambles A and B (columns), two possible outcomes (Out 1 and Out 2) and their probabilities (rows). The five main columns represent the different categories of the decision task. Each category consists of eight different decision tasks that are presented in rows. The expected value for each pair of gambles is approximately the same. All gambles are coded, so that gamble A is predicted by the $\mathrm{PH}$ and, in each gamble, outcome 1 is lower than outcome 2 . In the presentation, however, the positions of gambles and outcomes were varied according to a fixed random design. 


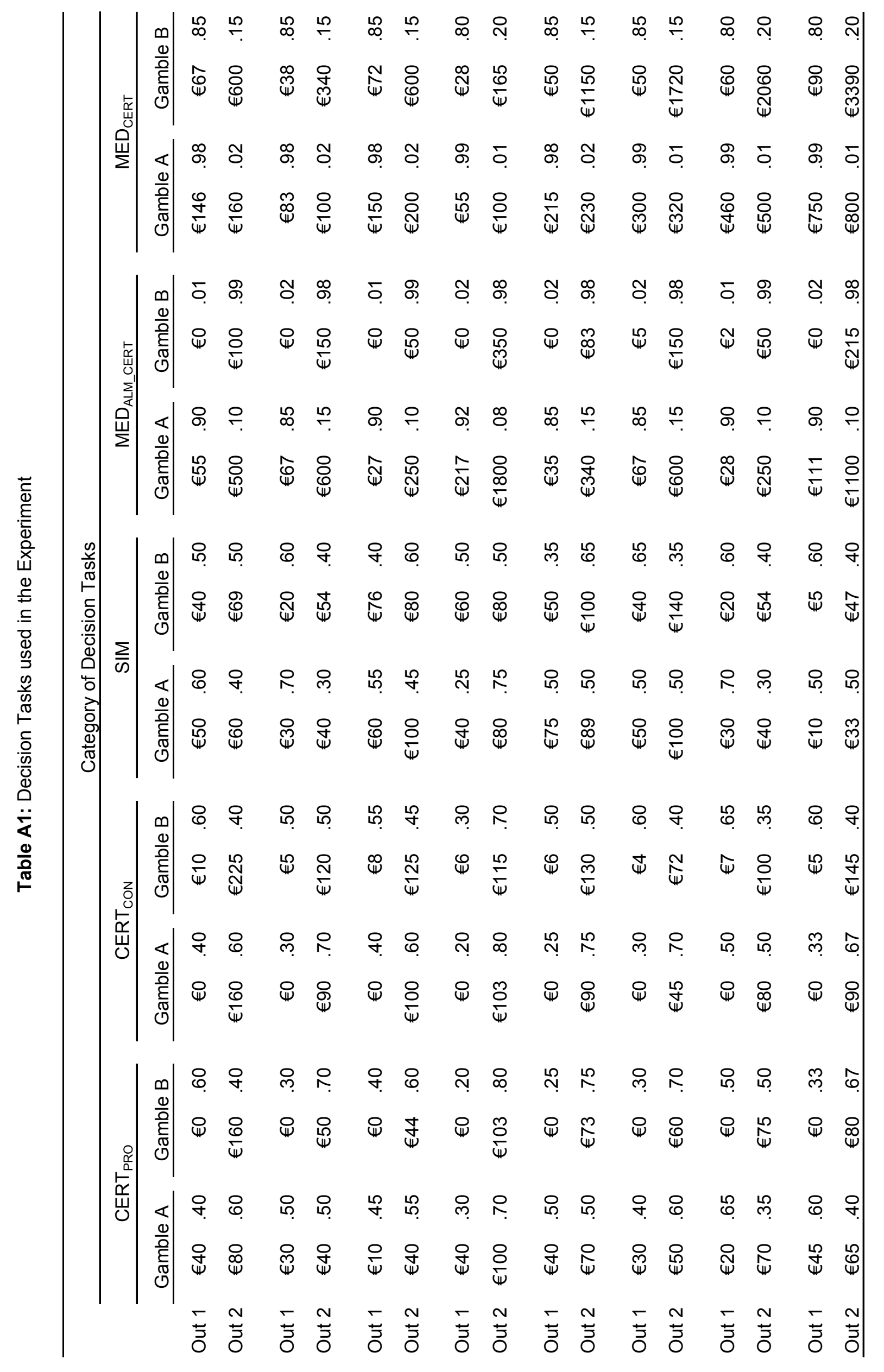




\section{Authors' Note}

Andreas Glöckner, Max Planck Institute for Research on Collective Goods, Research Group Intuitive Experts, Bonn, and Ann-Katrin Herbold, University Hospital Bonn, Department of Psychiatry, Division of Medical Psychology.

Correspondence concerning this article should be addressed to Andreas Glöckner, Max Planck Institute for Research on Collective Goods, Kurt-Schumacher-Str. 10, D-53113 Bonn, Germany. E-Mail: gloeckner@coll.mpg.de.

\section{Biographical Sketches}

Andreas Glöckner is head of the interdisciplinary research group Intuitive Experts at the Max Planck Institute for Research on Collective Goods, Bonn. His research focuses on the cognitive processes underlying judgment and decision making, parallel constraint satisfaction models, methods for measuring intuition, and in experts' decision making. He also does interdisciplinary research on legal and economic issues.

Ann-Katrin Herbold studied psychology at the universities of Erfurt and Frankfurt (Germany), and at the University of Exeter (England). She reached her master's degree in 2007. Currently she is working as a PhD student and research assistant in the EU project "PERCEPT - Perceptual Consciousness: Explication and Testing" at the University Hospital Bonn. 